\title{
Review Article \\ Properties of Specialist Fibres and Bragg Gratings for Optical Fiber Sensors
}

\author{
John Canning \\ Interdisciplinary Photonics Laboratories, School of Chemistry, University of Sydney, Sydney, NSW 2006, Australia \\ Correspondence should be addressed to John Canning, j.canning@usyd.edu.au \\ Received 4 March 2009; Accepted 9 June 2009 \\ Recommended by Christos Riziotis

\begin{abstract}
The advent of optical fibres based on air holes running along their entirety opens up new directions in addressing various properties relevant to sensing, including the temperature/strain challenge of optical fibre sensors. This paper looks at the measurement challenges associated with temperature and strain, examines the potentially unique functionality structured fibre designs with and without gratings open up, and briefly describes some current research directions within conventional fibre and grating technologies.
\end{abstract}

Copyright ( $\odot 2009$ John Canning. This is an open access article distributed under the Creative Commons Attribution License, which permits unrestricted use, distribution, and reproduction in any medium, provided the original work is properly cited.

\section{Introduction}

The projected market size of optical fiber sensors generally is expected to exceed \$US 1.6 billion in 2014, up from the 2007 figure of \$US 235 million [1]. Grating technologies are increasingly pervasive within this market finding applications ranging from biomedical, chemical, and cosmetic [2-6] to structural health monitoring (SHM) of buildings, vehicles, aircraft, bridges, trains, and windmills [7-19]. Two important characteristics dominate the criteria of gratings for sensing applications: temperature and strain/pressure sensitivity, both of which are affected by the type of optical fibre and the performance parameters not only of the grating itself but also of the type of fibre and the required packaging.

Traditionally, the separation of the intertwined temperature and strain response has been done using reference grating elements to calibrate out one or the other parameter. However, innovations in new fibre design, largely centred on structured fibre technology [20], offer radically new solutions that may circumvent the need for secondary reference gratings. The ability to tailor the hole distribution in general allows an incomparable flexibility in fibre design and in parameter control, not just of temperature and strain but of many others. For example, by adjusting the crystal lattice parameters of a photonic crystal fibre appropriately, unique composite system properties providing multiple functions, such as dual dispersion compensation and Raman amplification [21], are possible. By controlling the hole distribution and size, mechanical (including acoustic and pressure response) properties are altered as well as even more basic properties such as strain optic coefficient. Inserting material gives greater scope for adjusting composite system properties such as the effective thermo-optic coefficient of zero, ideal for zero temperature gratings $[22,23]$. The recent demonstration of microfilling a structured optical fibre with three separated laser dyes around the core of the fibre [24] reveals the full potential of structured fibres, particularly bringing the speculative prospect of the "labin-a-fibre" [25] closer to reality. Grating technology will inevitably contribute to such functionality-it will, as noted, also benefit the separation of temperature measurements with the grating, the most immediate area where practical progress using structured optical fibre gratings will likely be seen.

\section{Structured Optical Fibres and the Evanescent Field}

Figure 1 illustrates three contemporary examples of interest to the sensing community: the first is a single mode "photonic crystal fibre" [27] (so-called because of the periodic lattice although diffractive effects are clearly observed at short 


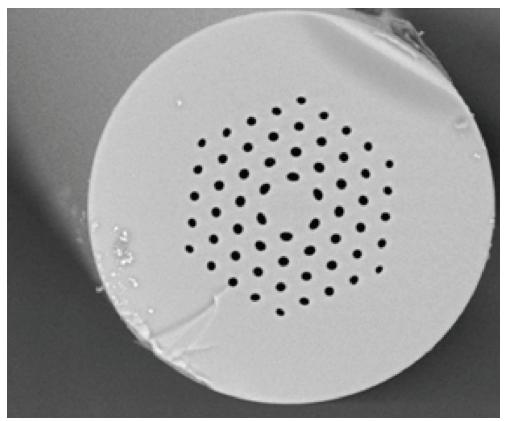

(a)

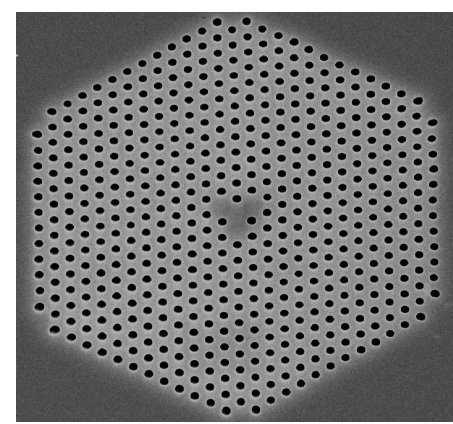

(b)

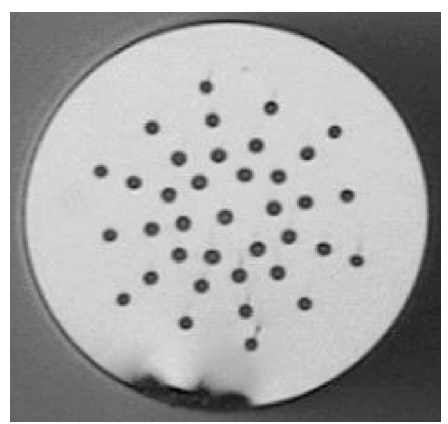

(c)

Figure 1: Examples of common structured optical fibres into which gratings have been written: (a) low loss $(<4 \mathrm{~dB} / \mathrm{km}) 4$-ring all-silica photonic crystal optical fibre; (b) 12-ring photonic crystal fibre with triangular core and photosensitive, high NA step-index germanosilicate centre; and (c) a simple Fresnel fibre.

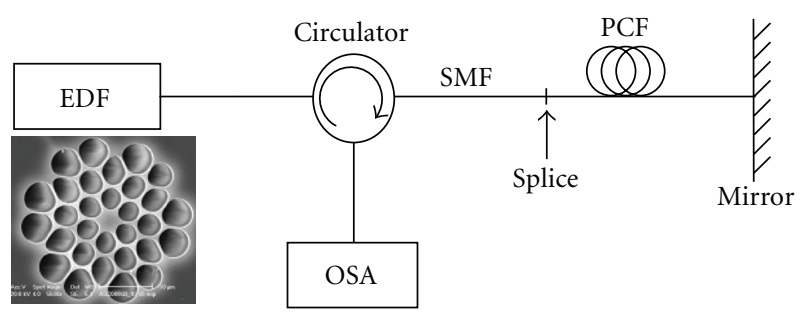

(a)

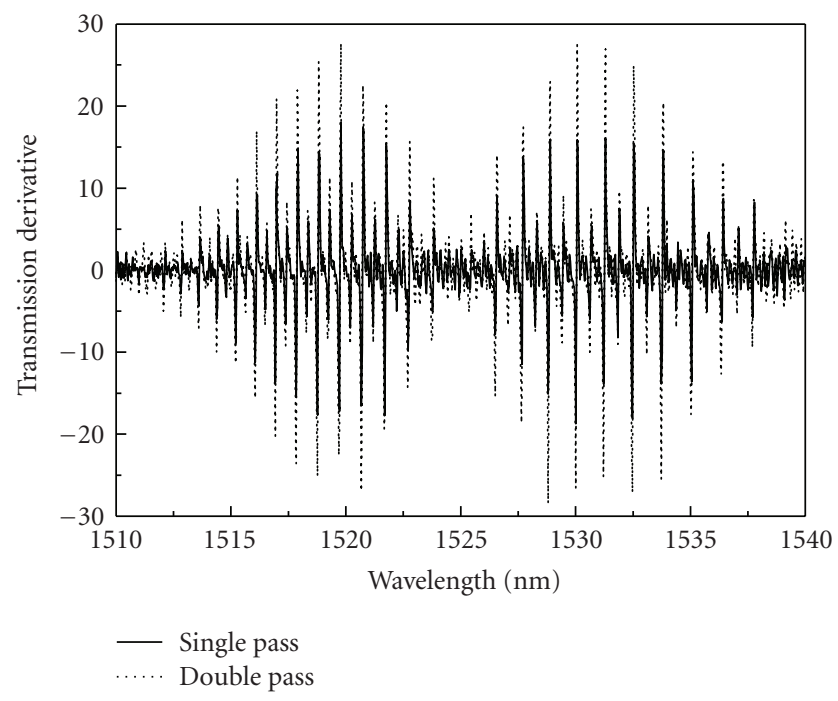

(b)

FIGURE 2: All-fibre add-drop acetylene reference cell using a photonic crystal fibre (a). Spectra for both single pass and double pass of the cell are shown (b) [26]. (EDF-erbium doped fibre source, OSA-optical spectrum analyser, SMF-standard single mode fibre smf 28, PCF-photonic crystal fibre: scanning electron microscope image of cross-section shown above $y$-axis).

wavelengths-see, e.g., $[28,29])$; the second is a photonic crystal fibre with a highly nonlinear and photosensitive core surrounding by a triangle of silica which supports an unusual additional mode, and the latter is a Fresnel fibre [30], where propagation is diffractive and the mode peak intensity is

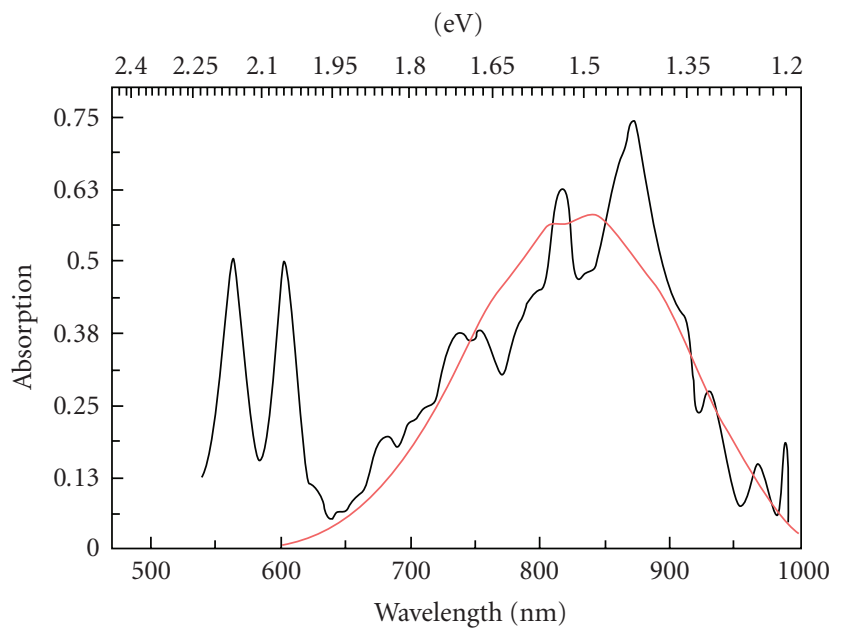

Figure 3: Absorption measurement of PCF containing a porphyrin thin film deposited on the surfaces of its holes in DMF: (length = $50 \mathrm{~cm}$, absorption rescaled to $25 \mathrm{~cm}$ ) the typical Q-band (559 and $599 \mathrm{~nm}$ ) is observed along with a new near-IR band (660-930) nm. Numerical simulation is shown in red for a typical charge transfer band. More details can be found in [5].

surprisingly well localised in the hole centre. Perhaps more interesting still, light is partially focused at the output [3032], from which small phase zone plates can be fabricated [33].

An important consideration for exploiting the structure within these fibres is the penetration depth, and overlap integral, of the evanescent field within the holes. Within a conventional photonic crystal fibre (a structured fibre with a regular periodic array of holes determining the cladding without necessarily having significant diffractive effects at the sensor probe wavelength), the evanescent field overlapping with the holes is a key determinant of the effective interaction lengths possible. A typical "endlessly single mode" fibre usually has an overlap integral that is too low to be of significant use so the air fraction tends to be increased. Generally, a few per cent overlap at $1500 \mathrm{~nm}$ becomes useful; the figure increases towards the red and decreases towards 


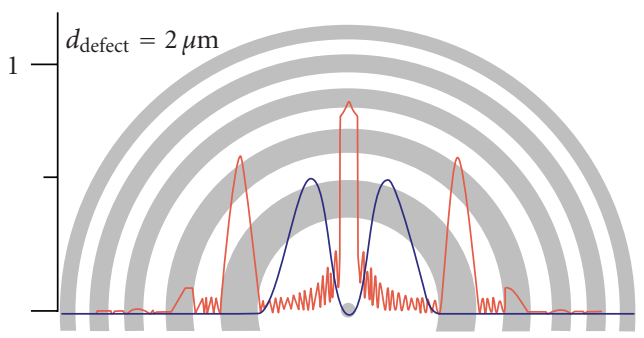

(a)

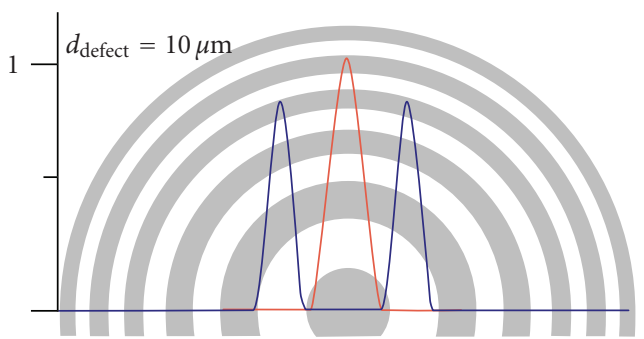

(c)

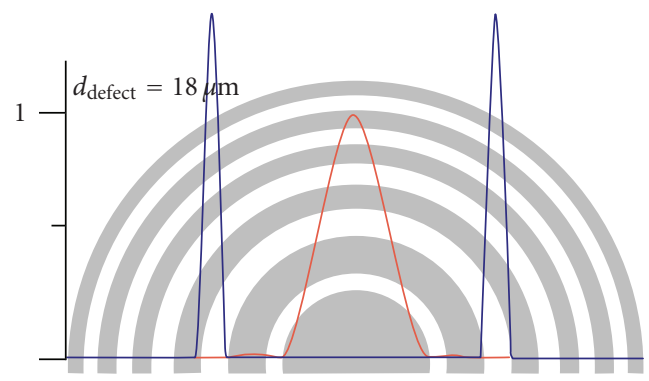

$\begin{array}{lll}- & \text { Air-guided mode } & \text { Air } \\ & \text { Silica-guided mode } & \square\end{array}$

(e)

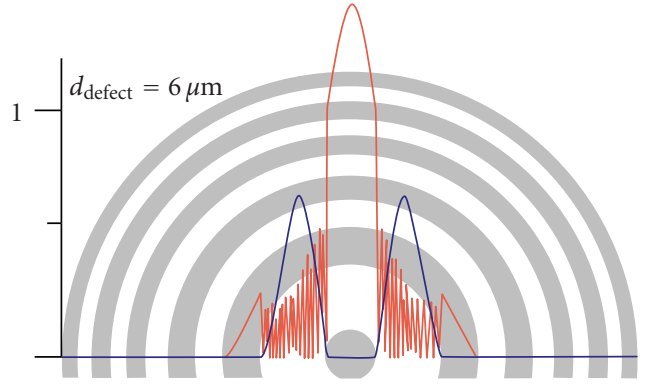

(b)

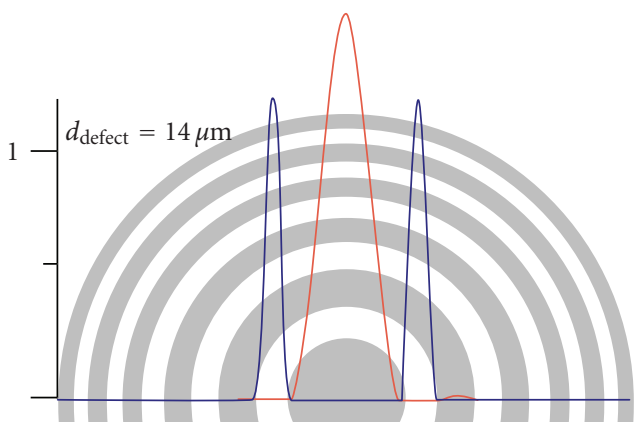

(d)

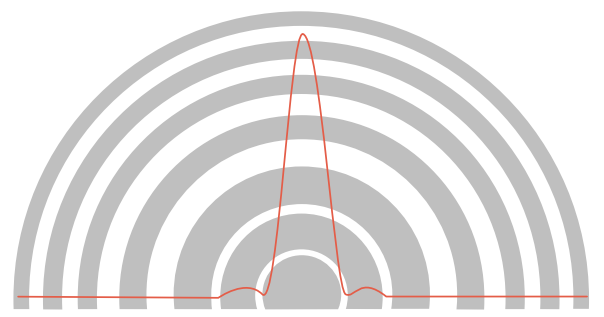

$\begin{array}{lll}- \text { Air-guided mode } & \text { Air } \\ - \text { Silica-guided mode } & \text { Silica }\end{array}$

(f)

Figure 4: (a)-(f). Numerical simulation of (red) optical localisation in varying hole size within a bandgap Fresnel fibre and (blue) optical localisation within the silica. As the hole size becomes smaller ( $\mathrm{a}$ and b), a step-like discontinuity arising from the interface boundary is observed [34]. There appears to be enhanced field localisation of the evanescent field in addition to that expected from the far-field phase diffraction of the Fresnel structure.

the blue. Notably, the bulk of the evanescent field lies within the first $60-100 \mathrm{~nm}$ from the surface-it is often not recognised that the probing field is therefore highly concentrated close to the near field regime, making it an ideal tool for exploring interface effects but also a potentially problematic issue when comparing to bulk references. On this scale what sometimes appears to have no detectable interaction with a silica surface in free space can sometimes be picked up over long interaction lengths complicating any analysis that relies on bulk sample, or indeed short path length, interactions. Therefore, whilst for many species this is not a problem, for others, particularly those associated with biodiagnostics where surface interactions are not unexpected, the issue may be both problematic and opportunistic. For example, to highlight this point, an overlap of a few percent is sufficient to perform numerous sensing and device experiments. Figure 2 shows a double-pass add-drop all-fibre acetylene gas reference cell made with a photonic crystal fibre, filled with low pressure acetylene, and with a few percent field overlap with the first ring of holes in the near IR [26]. A crosssection of the fibre is shown for comparison with Figure 1(a). In this case, there is no observable difference between these measurements and bulk, consistent with the expectation that acetylene does not react with the glass surface. On the other hand, an identical fibre was also used to make the first direct observation of charge transfer between porphyrins and silica [5], a molecular interface effect which has not been previously observed. This added effect generates a large 


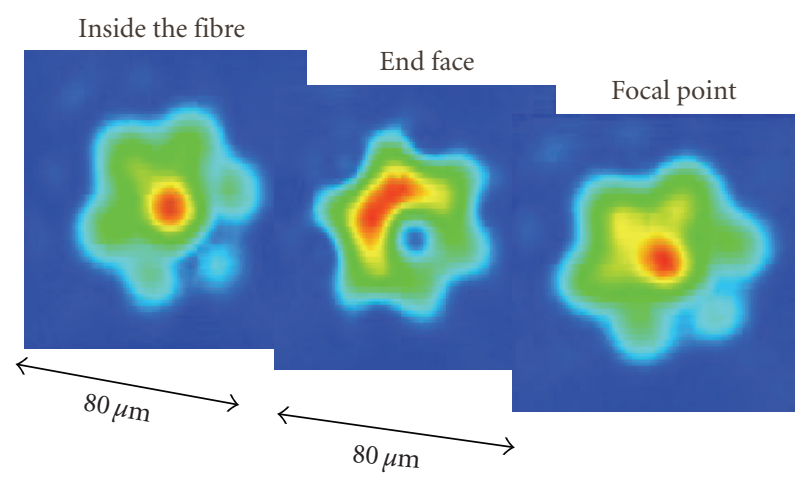

Figure 5: Near field profile of the end of the Fresnel fibre shown in Figure 1(c). The centre profile is the end face, and the other profiles are imaged using an objective lens within and beyond the fibre, respectively. Complex interfering supermodes are observed including one with tight optical localisation within the hole.

near IR band (Figure 3) which needs to be considered if porphyrins are to be used in biodiagnostics that operate in this region, for example.

A solution for writing gratings is to introduce a photosensitive core, as in the second example illustrated in Figure 1(b). This is often done by preparing a standard optical preform using MCVD and then etching out the core (typically close to $1 \mathrm{~mm}$ in diameter) before inserting into the structured optical fibre preform. Whilst this process clearly introduces a standard photosensitive core and makes grating writing easy, the benefits of single-material fibres are lost and the added complication of further confinement away from the holes by a step index needs to be dealt with. This latter problem can in part be handled by making the photosensitive region much smaller than the effective mode field diameter and, consequently, such fibres often have smaller cores.

Numerical simulation of a Fresnel fibre with decreasing hole size in the centre that showed a highly localised peak intensity within the hole is possible once subwavelength dimensions were reached [34]. This localisation arises as a result of the impedance matching required at the interface between two materials of different refractive indices and on the lower index side the electric field, $E$, scales as the square of the ratio of the index contrast, $n_{1} / n_{2}$ ( $E$ proportional $\left(n_{1} / n_{2}\right)^{2}$. The higher the index contrast the more significant the effect-in silicon photonic crystal waveguides this forms the basis for very sensitive nanoparticles detection and biosensing on a chip [35-38]. Figure 4 shows the simulation for the Fresnel fibre. In any case, optical localisation over the hole is observed experimentally at larger sizes attributed to the diffractive properties of the Fresnel fibres [30-32]. Other work also supported very tight optical localisation within a silica structured photonic crystal fibre using a subwavelength hole [39]. All these forms of optical localisation phenomena have significant potential for enhancing sensitivity in many applications that require small volumes and high interaction with optical fields, such as biodiagnostics applications. For example, localisation of light is central to developing metal free optical microscopy both in the far and near fields [40].
Figure 5 shows the near field profile of experimentally observed localised light within a Fresnel fibre (Figure 1(c)) with a central air hole which is smaller than the effective mode wavelength. Also observed is the ring mode associated with the silica ring region around the central hole, as expected from simulation. There is evidence that the two localised propagating fields (modes) interfere every $80 \mu \mathrm{m}$ within this fibre sample. The localization of the evanescent field within the central air hole is difficult to observe since the resolution is diffraction limited. In contrast to so-called "photonic bandgap" fibres, a key advantage of this type of optical field is that there remains sufficient silica in the surrounding ring into which useful gratings can be written (shown later). (These "photonic bandgap" fibres are essentially diffractive fibres with only a partial bandgap; the index contrast is usually too small to have anything more than a small angular stopgap, which is analogous to diffractive fibres such as Bragg and Fresnel fibres.) These gratings can therefore be used to perform additional spectrally selective monitoring of samples probed by the high field within the central hole (in contrast to the much poorer overlap in the cladding holes). Quasicrystal fibres such as Fresnel fibres rely on simplifying the design based on the circular nature of the waveguide. Periodic lattices associated with 3D structures are unnecessary - instead designs based on omnidirectional filters allow much broader stopgaps. For example, the fibre in Figure 1(c) has a bandgap over $1000 \mathrm{~nm}$ wide [41]. These fibres also lead to significantly lower losses since the resonant coupling between interstitial regions (both in terms of optical field within and or at the interface of the glass regions, perhaps as surface modes) is reduced, as discussed later.

From the few examples illustrated above, the potential and challenges of structured optical fibres, which can generate complex propagation, can clearly benefit by the inscription of wavelength selective components such as Bragg gratings. Grating writing, therefore, within nonphotosensitive, pure silica, single material fibre becomes of paramount importance if many of these unique features are fully retained. In this particular paper, the focus is on specific silica-based Bragg grating technologies that address new directions in the temperature/strain challenge of optical fibre sensor gratings in conventional and structured optical fibres. This will serve to highlight the potential to sensing.

\section{Temperature and Strain}

Grating sensors rely on a shift, $\Delta \lambda_{B}$, in the resonance wavelength, $\lambda_{B}$, arising from either strain, $\varepsilon=\Delta l / l$, and/or temperature, $T$. This is because $\varepsilon$ affects a change in resonance through both spatial changes in period, $\Lambda$, and refractive index, $n$, whilst $T$ largely affects a refractive index change (although this is also affected by changes in stress between the core and cladding as a result of different thermal expansion coefficients, which in turn affects the stress contribution to refractive index change). In general, the relationship which binds strain and temperature with the grating resonance wavelength can be described simply in 
one equation (see, e.g., [42]):

$$
\frac{\Delta \lambda_{\mathbf{B}}}{\lambda_{B}}=\frac{\Delta n}{n}+\frac{\Delta \Lambda}{\Lambda}=(1-\rho) \varepsilon+\kappa T,
$$

where $\rho$ is the elasto-optic coefficient of the fibre, and $\kappa$ is the thermo-optic coefficient. The particular expression for studying specific stain parameters introduced by pressure can be obtained by noting Poisson's ratio and expanding the series if necessary. This expression forms the basis for nearly all Bragg grating-based optical fibre sensor applications.

\section{Conventional Step Index Fibres}

For nearly all practical cases using conventional silicatebased optical fibres, it is assumed that the fibre parameters are approximately those of pure bulk silica, which are well known $\left(\rho \sim 0.22, \kappa \sim 5 \times 10^{-6}\right)$. If the tolerable experimental error bars are sufficiently large, or a self-calibration reference is implemented for each sensor, then this is an acceptable approximation. However, it is just that-in reality, the actual response profiles that are obtained cannot be fitted so nicely by such a simplification-estimates of both strain optic and thermo-optic coefficients based on optical fibre work therefore often vary noticeably not only from bulk measurements but also between laboratories. This may come as a surprise, but this variation cannot be readily calibrated out using a universal standard-each fibre is genuinely different depending on how it was fabricated, by whom (an element of black magic sometimes comes into play!), what dopants are employed, and sometimes by what lathe system is used. In practice, there is an additional term that affects the values to (1) - frozen in mechanical pressures and strains. For instance, the axial strain generated by the fibre drawing process alone can be described as $[43,44]$

$$
\sigma_{z} \approx \frac{F}{2 \pi}\left[\frac{\eta}{\int_{0}^{R} \eta(r) r d r}-\frac{E}{\int_{0}^{R} E(r) r d r}\right],
$$

where $F=$ drawing force/tension, $h=$ viscosity, and $E$ is the Young's modulus. This strain increases with drawing tension, viscosity, or decreasing $E$. Consequently, the processing history becomes important in determining the optical fibre's mechanical properties, which are in turn dependent on the type and quantity of dopants used. These dopants also clearly affect the local refractive index of the core and, therefore, the confinement of the optical mode which in turn affects the measurands.

For most normal optical fibres made from germanosilicate, for example, optical fibre drawing conditions are selected that happen to produce a certain range of tensile stresses arising from the fact that conventional fibres are composite systems determined by two differing glass compositions between core and cladding. It is entirely possible to obtain the reverse-mechanical stress can be of opposite sign and exceed magnitude of thermal stress and lead to compressive stress at the interface depending on preparation conditions, impurities, and dopants $[45,46]$. For example, under very high drawing tensions, compressive stresses can be generated.

Equally important to the frozen-in mechanical stresses are the subsequent thermo-elastic stresses arising from differences in thermal expansion coefficient between core and cladding. The expansion coefficient for $\mathrm{SiO}_{2}$ is typically $\sim 0.55 \times 10^{-6}$ but this can be changed substantially by adding dopants. Adding $20 \mathrm{~mol} \%$ of $\mathrm{GeO}_{2}$ increases this to $2.8 \times 10^{-6}$ whilst adding only $12 \mathrm{~mol} \%$ of $\mathrm{P}_{2} \mathrm{O}_{5}$ increases this to $2 \times 20^{-6}$. This changes the temperature dependence of the fibre and in combination with the effects of frozen-in mechanical stresses means even fibres with identical recipes can vary between each other substantially. Table 1 summarises some of the thermal expansion coefficients, $\alpha$, per mol \% of common dopant added [47]. Interestingly enough, using materials with large negative expansion coefficients in sufficient concentrations can open up pathways to designing glass hosts that have reduced, zero or negative temperature dependence, an alternative to subsequent mechanical tensile stressbased packaging of optical fibre Bragg gratings currently sold commercially. Not considering the frozen-in mechanical stresses, only $5 \mathrm{~mol} \%$ of $\mathrm{TiO}_{2}$ is required to cancel out the positive temperature dependence of $\mathrm{SiO}_{2}$. Of course, an important consideration is whether the dopant can be readily introduced and whether it will impact on the desired properties of the optical fibre, including photosensitivity. For this latter case, the almost ubiquitous material chosen is $\mathrm{GeO}_{2}$ and often codoped with $\mathrm{B}_{2} \mathrm{O}_{3}$ to facilitate better mixing into the silica glass network and to lower the refractive index step. Standard grating writing within these fibres takes advantage of highly coherent frequency doubled $\mathrm{Ar}^{+}$lasers to generate arbitrary grating profiles using a computer controlled free space interferometer. This level of sophistication was developed for the telecommunications industry where alternative filters to etalon-based thin films were needed to achieve superior performance. Inevitably, such technology would find applications in chemical sensing diagnostics since a filter designed to filter out a specific molecular absorption or emission band can be readily made. This has been particularly useful already for those species with overlapping bands in existing telecoms windows, such as $\mathrm{OH}$ a problematic source in spectroscopic studies of the night sky. Fibre grating filters promise to displace current thin film etalon filters [48], for example.

For active sensor devices based on rare earth ions, it is often preferable to avoid these dopants since they have a deleterious impact on the longevity of the upper level lifetimes crucial for laser and amplifier performance, particularly in codopeded systems such as $\mathrm{Er}^{3+} / \mathrm{Yb}^{3+}$ that are more exposed to phonon decoupling. For these, preferable dopants include $\mathrm{P}_{2} \mathrm{O}_{5}$ for reduced decoupling of the upper level excited states although at the expense of higher scattering losses and lower fibre thermal tolerance. For higher power laser operation and power handling generally, $\mathrm{Al}_{2} \mathrm{O}_{3}$ is the preferred dopant. Mixing and phase separation are two important considerations when optimising optical fibre functionality because the issue of loss is critical for most 
TABle 1: Thermal expansion coefficients (per degree Celsius) for common glass dopants used in conventional optical fibres.

\begin{tabular}{lc}
\hline Dopant & $\alpha\left(\times 10^{-6} / \mathrm{mol} \%\right)$ \\
\hline $\mathrm{P}_{2} \mathrm{O}_{5}$ & 1.3 \\
$\mathrm{GeO}_{2}$ & 0.7 \\
$\mathrm{~B}_{2} \mathrm{O}_{3}$ & 0.7 \\
$\mathrm{~F}$ & -0.02 \\
$\mathrm{TiO}_{2}$ & -1.0 \\
\hline
\end{tabular}

functional applications, especially long distributed sensor systems that consist of numerous components.

Another important dopant that has not been considered in detail in the past (at least with respect to grating writing) is $\mathrm{OH}$ impurities. $\mathrm{OH}$ impurities are known to give rise to compressive stresses $[45,46]$. It is almost certain that the formation of $\mathrm{OH}$ acts to create an internal pressure within the glass that can help offset tensile stresses, if not exceed them. The ramifications for a well-known photosensitive enhancing procedure-hydrogen loading-are significant. $\mathrm{OH}$ formation is often a by-product of conventional grating writing and its formation conveniently accounts for the induced index changes through Kramers-Kronig analysis [49]. The effect of mechanical stress within the fibre is not widely considered. On the other hand, it seems highly plausible that this mechanical effect contributes substantially to the total index change possible by alleviating the stresses that might otherwise restrict normal type I grating formation (where stresses have been observed to increase directly at the core-cladding interface $[50,51])$. Furthermore, it seems crucial that this effect takes place for type I $p$ (or type IA) positive gratings [52]. There is no doubt that the complexity and range of grating writing opportunities [52] across the type I spectrum are dependent on all these processing conditions and dopant properties. If this insight is correct, we can actually begin to make predictions of additional effects one might expect using other dopants, for example, $\mathrm{Cl}$ is also known to reduce the thermal expansion coefficient of the core $[53,54]$ and is a standard drying material used in MCVD optical fibre fabrication. If used appropriately, axial stress during fibre fabrication may be reduced. If standard germanosilicate optical fibres are considered, by reducing the total frozen-in stresses into the final fibre, it is likely that the photosensitivity of type I gratings can be substantially optimised, with potentially important outcomes such as improved thermal stability. Likewise, the impact on higher processed gratings such as negative index gratings and regenerated gratings bears consideration.

Clearly, in addition to changing the mechanical and thermal response parameters of a fibre Bragg grating, there remains substantial scope for tailoring the photosensitive response of conventional optical fibres. Conversely, there has been considerable work in tailoring the grating writing process itself to the medium-the advent of $193 \mathrm{~nm}$ laser applied to optical fibre Bragg gratings, in particular, extends the application to many other materials, including important phosphosilicate and aluminosilicate systems that benefit fibre lasers [59-61]. New lasers, especially femtosecond lasers

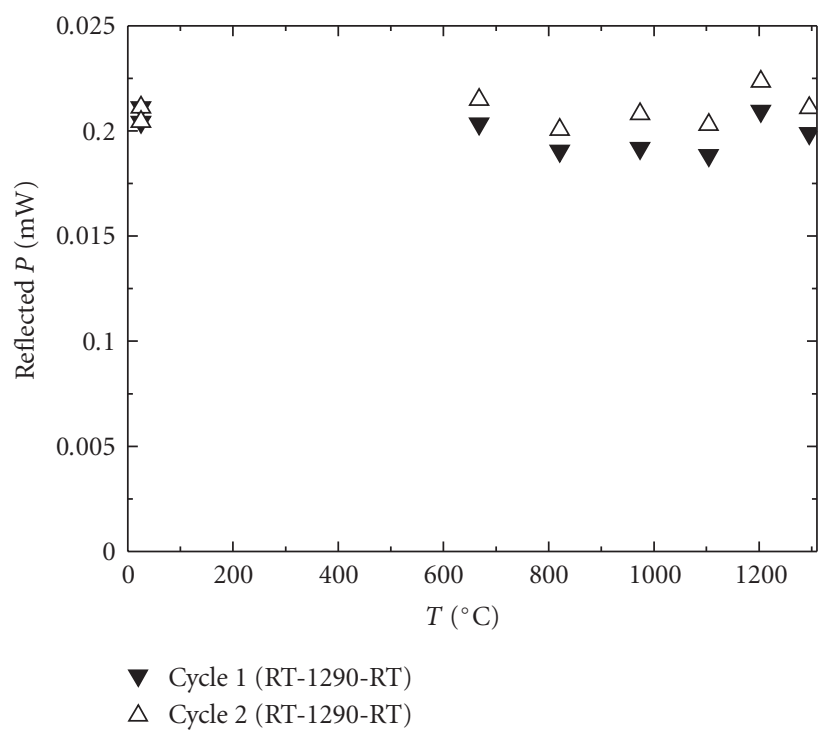

FIGURE 6: Temperature performance of a regenerated grating $(10 \% \mathrm{~T})$ in a telecom matched photosensitive germanosilicate core optical fibre. Two full cycles from room temperature to $1295^{\circ} \mathrm{C}$ are shown [55].

that depend less on the dopants and more on the damage threshold to affect index change $[62,63]$, promise to have a similar impact. Below the damage threshold, the exploration of photosensitivity into other materials for conventional fibre Bragg gratings has meant that in parallel a large number of new processing recipes allow the properties of Bragg gratings themselves to be tailored substantially-for a review of these photosensitive mechanisms see [52].

Most conventional fibres, therefore, have an inbuilt stress (or pressure) that adds or detracts to the actual sensitivity of the component. The strain and temperature curves as a function of applied pressure will in all likelihood vary across fibres from recipe to recipe and composition to composition, a problem that raises interesting challenges for the definition and measurement standards, international bodies must eventually determine if there are to be universal generic sensor elements. Differences between laboratories are often attributed to arising from variable experimental parameters rather than, potentially, from differences in the mechanical and thermal parameters between fibres.

4.1. Extreme Gratings. As an illustration how conventional optical fibres remain far from exhausted in terms of novel research and direction for grating sensors, and how little is still understood in terms of the underlying photosensitive mechanism of even the most thoroughly investigated type I gratings, it is worth examining recent reports of gratings in conventional fibres that survive repeated cycling at ultrahigh temperature $\left(>1000^{\circ} \mathrm{C}\right)[55,64,65]$. These socalled regenerated gratings presently outperform all other gratings - despite the expansive growth in new writing recipes and formulations, these results were obtained by taking ordinary type 1 gratings and thermally processing them with an appropriate recipe where the temperature 


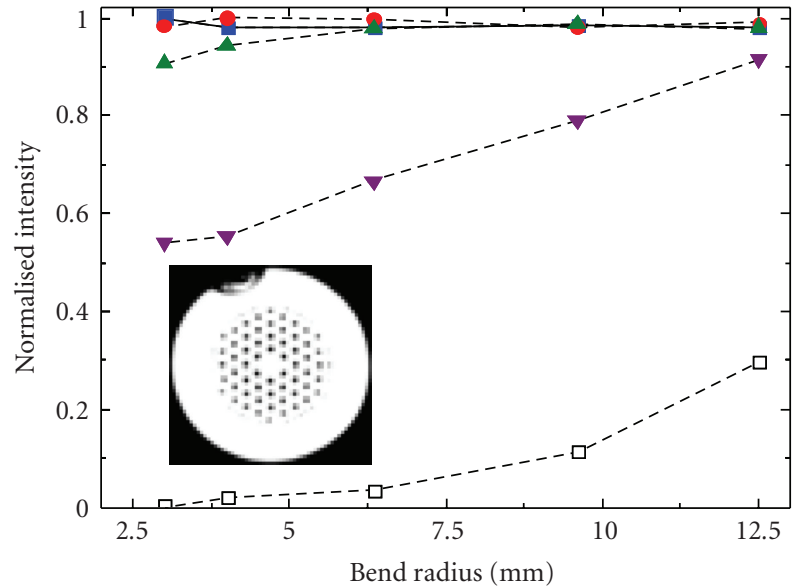

(a)

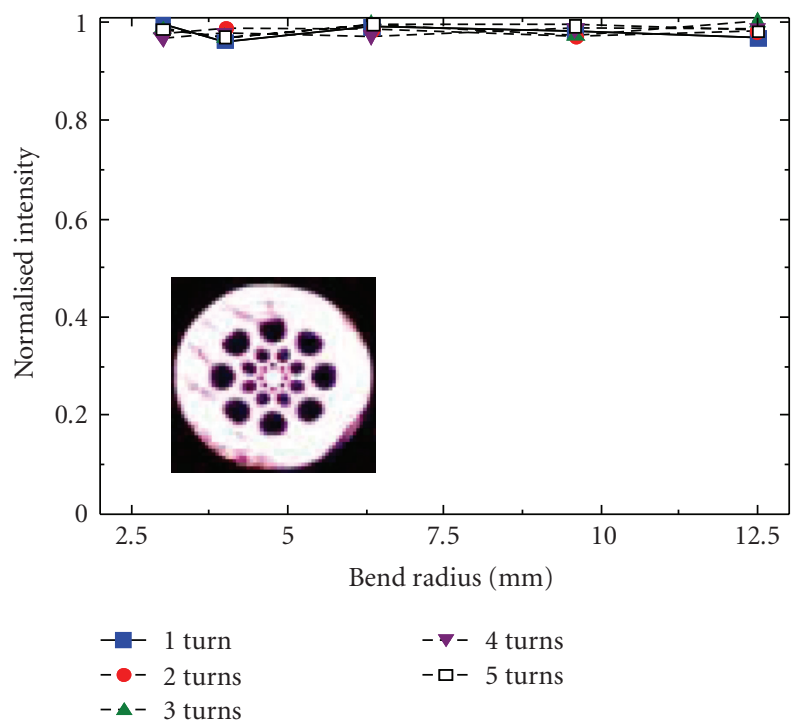

(b)

Figure 7: Observed bend loss as function of bend radii for (a) regular photonic crystal fibre and (b) "fractal" (or chirped Fresnel) fibre [56].

is ramped to $\sim 9000^{\circ} \mathrm{C}$ and the regenerated grating allowed to evolve. The final regenerated $5 \mathrm{~mm}$ long grating can be as strong as $25 \%$ transmission and can be cycled repeatedly as high as a temperature of $1295^{\circ} \mathrm{C}$ with full recovery at room temperature [55], as illustrated in Figure 6. The grating can outlast the fibre itself, that is, whilst the fibre becomes brittle at such temperatures and often breaks, no observable impact on the grating spectra is noted. A simple diffusion model alone, based on hydrogen bonding and oscillating reactions, therefore, seems unlikely to suffice to explain these results. $\mathrm{H}$ is crucial for any diffusive process, particularly via $\mathrm{OH}$ transfer. One argument rests on the formation of molecular water as a mean of displacing oxygen, which would not normally be possible [64], based on a modified oxygen diffusion model [66]. However, such a transfer implies that substantive oxygen-deficient silica is created, which would likely lead to a crystallisation of

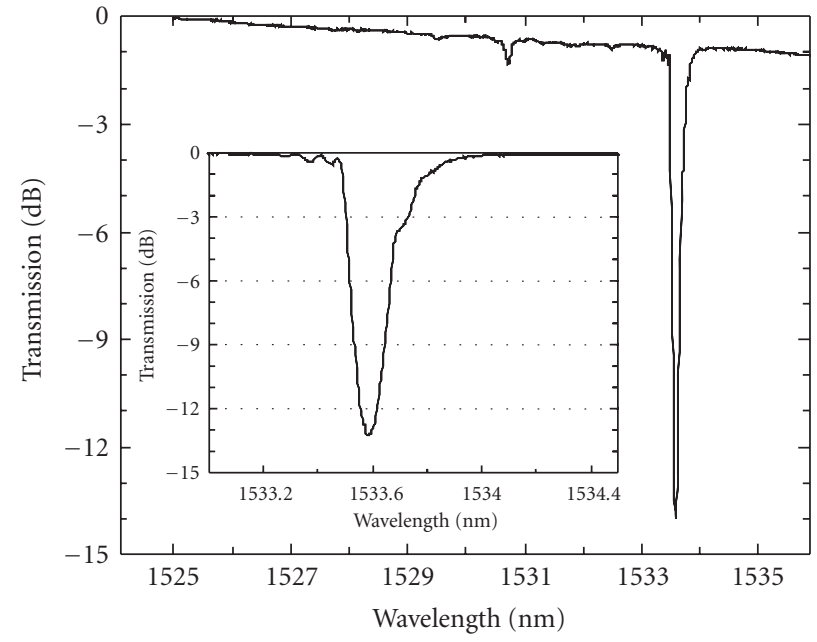

FIGURE 8: Grating in pure silica single material photonic crystal fibre [57]. Some evidence of birefringence $\left(<\times 10^{-5}\right)$ is observed in this example.

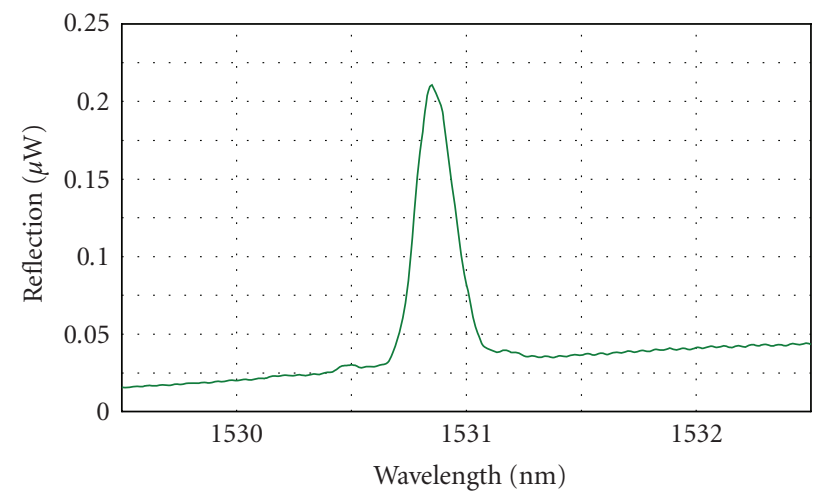

FIGURE 9: Reflection spectra of a $5 \mathrm{~dB}$ strong grating within the Fresnel fibre shown in Figure 1(c) [58]. The optical intensity of a propagating mode peaks in the core hole.

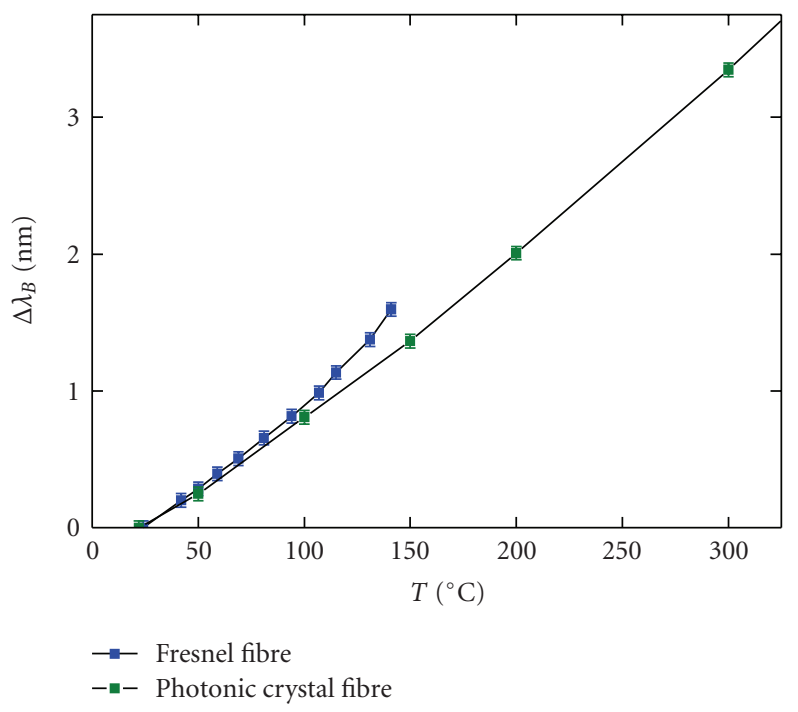

Figure 10: Temperature dependence of the fundamental mode within two types of structured optical fibre [58]. 
silicon or structural transformation to a larger bond angle polymorph, both of which would give rise to large scattering and large index changes. Alternatively, the seed grating with hydrogen allows a different glass quenching and relaxation to occur in the processed regions that give rise to, perhaps, a crystallised or amorphous grating structure of similar tetrahedral properties. This remains the subject of ongoing work. Nevertheless, these gratings can now be applied to ultrahigh temperature applications including temperature monitoring of smelters, furnaces, and engines.

\section{Single Material Structured Fibres}

Equation (1), along with the discussion above, would suggest that single material optical fibres will have much less variability to parameters and, in particular, the effective thermooptic coefficient should be lower. The possibility that such fibres might form the basis for more reproducibility between laboratories, amongst many other applications, makes the idea of single material fibres especially attractive. This reduced temperature dependence of such fibres has recently been verified many times in many ways, not only with Bragg gratings, but also for long period gratings in single material photonic crystal fibres [67] and, particularly attractive, linearly and spun birefringent photonic crystal fibres and devices utilizing them [68-73]. Modal interferometers using a short length of single material photonic crystal fibre [74] have also been used as a temperature insensitive strain sensor [75]. Specifically, structured optical fibres based on air holes running along their entire length have one other key advantage not possible with conventional fibre technology based on dopants. Essentially, step index propagation can be achieved by a reduced average cladding index determined by the distribution of air holes.

The structured optical fibre is made up of so-called leaky modes where the normal confinement boundaries defining modes in convention step index fibres are less defined because there is leakage between the holes. In perfectly periodic lattices this leakage translates into coupling between the interstitial regions, both in terms of optical field within the region and the optical field at the interface (sometimes described in terms of surface modes), and the surprising possibility of having complex cladding supermodes with close to equal or, in some cases, higher effective index than the core mode. In fact, this problem is almost certainly the reason why in terms of low loss fibres one would not use a periodic structure. Bend loss in particular is significantly worse for a periodic lattice fibre than even conventional step-index fibre with substantially lower NA (this beggars the explanation of what NA means in such fibres?). On the other hand, by removing the periodic lattice and preventing interstitial optical coupling of light, quasiperiodic or other nonperiodic Fresnel and "Fractal" fibre designs have been shown to reduce bend loss so effectively that they outperform conventional fibres. Zero bend loss is possible [56], as shown in Figure 7. For many sensor and other device applications this is a highly desirable property and allows tight bending of optical fibres. This net result also shows fundamentally the difference between higher-order modes and fundamental modes within photonic crystal fibres-the loss differential can be huge.

Given that, in the first instance, the fundamental leaky mode of the structured fibre has a $k$ vector along the fibre axis and therefore the role of leakage loss is somewhat suppressed, the fundamental mode may not be so distinctive in behavior to that of the step index analog. This forms the justification for the step-index approximation of simple structured fibres where the cladding index is, on average, lower than the core index. In contrast, when higher-order modes are present they will be significantly affected by leakage loss and greater access to the evanescent field within the holes-a property which opens up new opportunities for sensing applications. For example, because the difference between index and loss is large between the fundamental and higher-order mode of a two-mode structured optical fibre, it is possible to exploit this to create an internal reference for a Bragg grating. This was done using, instead, an active grating structure in the form of a DFB fibre laser [76]. Equivalent intermodal interference within photonic crystal fibres has also been demonstrated [74], and this needs to be considered when short devices are fabricated.

The ability to write gratings in single material fibres was first demonstrated using $193 \mathrm{~nm}$ from an ArF laser [57]. A key challenge that needed to be overcome was the large attenuation of the writing beam through scattering of the lattice structure - a comprehensive description of these processes can be found in [77]. Figure 8 shows the first such grating in a regular photonic crystal fibre (similar to that in Figure 1(a)). An immediate advantage is the absence of cladding modes since the index is not confined solely to the core. For conventional fibre technology, a photosensitive matched cladding needs to be custom designed to achieve a similar result. Figure 9 also highlights another important example - a $5 \mathrm{~dB}$ strong grating within a Fresnel fibre [58]. Although the optical mode has a large amount of optical filed interacting within the hole, the fibre design is such that there is sufficient propagation within the silica ring region into which the grating is inscribed so as to see the grating. By making the field smaller the optical localisation of evanescent light into the hole can be exploited for extremely sensitive biodiagnostics and chemical sensors.

\subsection{Temperature Response}

5.1.1. Fresnel Versus Photonic Crystal Fibre. Figure 10 shows the relative wavelength shift of a Bragg grating written by 2-photon excitation within a single material all-silica Fresnel fibre (Figure 1(c) and Figure 8) and a similar single material, single mode, all-silica 4-ring photonic crystal fibre (Figure 1(a)) as a function of temperature [58]. Below $100{ }^{\circ} \mathrm{C}$ the Fresnel fibre has a slightly higher temperature dependence, or effective thermo-optic coefficient, $\left(1 / n_{\text {eff } 1}\right)\left(\partial n_{\text {effl }} / \partial \mathrm{T}\right)=7.8 \times 10^{-6} /{ }^{\circ} \mathrm{C}$ compared to the photonic crystal fibre of $6.5 \times 10^{-6} /{ }^{\circ} \mathrm{C}$, although both are very close to that of pure silica $\sim 6 \times 10^{-6} /{ }^{\circ} \mathrm{C}$ [47]. There is a quadratic dependence on this effective thermo-optic 
coefficient, which is particularly pronounced for the Fresnel fibre. This is directly attributable to mode field confinement by the air holes as the index of the glass changes and, hence, great care must be taken when interpreting and comparing the temperature dependence between structured optical fibres. The apparent observation of a sharp transition within the Fresnel fibre may characterize a cut-off condition for propagation which is especially sensitive in the Fresnel fibre given the larger dependence on diffractive propagation. As noted previously, the role of surface optical field localization also remains unclear.

The illustrated results reflect, overall, huge scope to adjust both the fibre design and fibre parameters and control properties such as the temperature dependence. This can be applied to enhance various configurations of thermally tunable devices using structured fibres, active or passive, and gratings, for example. For sensing applications this control over the temperature dependence can be used to help separateing the intertwined relationship between temperature and strain. Single material fibres have other advantages, for example, given that the index contrast with air is sufficiently high, properties such as form birefringence can be readily introduced into these fibres. Form birefringence within a single material fibre is free of the temperature problem associated with two materials of different thermal expansion coefficient - fibre that has a zero temperature dependence of birefringence from -20 to $+800^{\circ} \mathrm{C}$ has been demonstrated [68]. These results were extended to a spun version of this fibre [72] which has important applications, amongst many, within electric field sensors and gyroscopes, where a critical parameter is packaging to isolate the system from temperature fluctuations. These two can be combined with Bragg gratings for enhanced and multifunctional properties.

5.1.2. Fundamental versus Higher-Order Modes. The stronger evanescent field interaction of the higher-order with the air holes can allow the fundamental mode to be used as a relative reference arm, an attractive all-in-one fibre solution. This possibility was recently extended to DFB fibre laser operation where lasing could be obtained on the fundamental mode or the higher-order mode, using a doped aluminosilicate core to further confine the fundamental mode away from the holes [56]. Such active grating structures allow enhanced resonance detection of many measurands, for example, it is an important approach to make optical fibres sufficiently sensitive for undersea acoustic detection [78, 79]. Below the lasing threshold, such a structure was used to study strain and temperature within structured optical fibres [80]. The effective indices of the two modes give rise to two distinct grating peaks (Figure 11). The modes are determined by the corresponding fractional powers $(\eta)$ of each mode within the higher index doped region (0.002) and in the surrounding silica. Leakage phenomena play an important part in determining and defining the mode field radius and, therefore, the fractional distribution of light of the modes either in the doped core or in the silica. Those modes with large transverse vector components will be sensitive to changes in the hole shape and stress between the holes in the

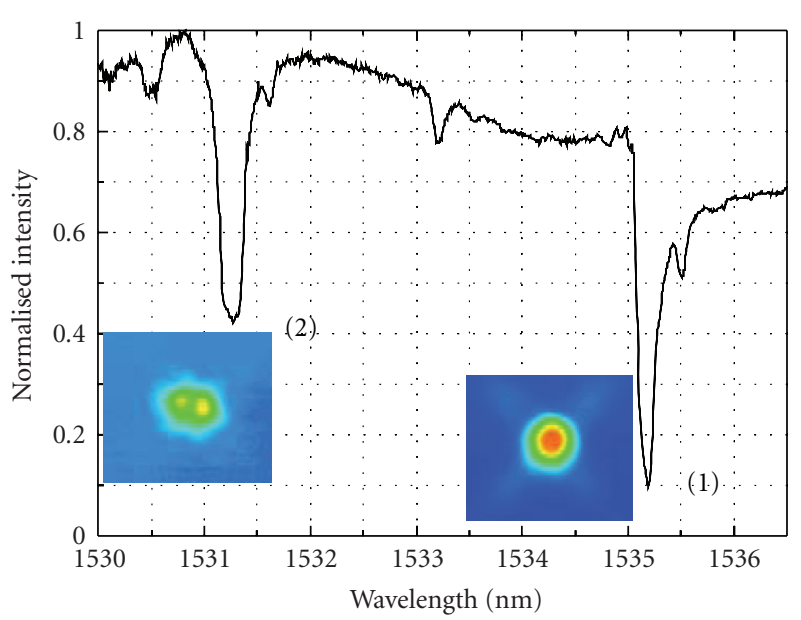

Figure 11: Transmission spectrum of a two-photon written Bragg gratings within a two-mode 4-ring photonic crystal fibre. (1)fundamental mode; (2)-higher-order mode and the dip in the centre is some cross coupling between the two arises from some asymmetry in transverse profile of the induced index change [80].

structure arising from, for example, tensile or compressive stress applied along the fiber.

But it is also clear that this sensitivity will depend on air fraction, the regularity of the lattice, the constituent materials, and the type of index guidance. For example, the Fresnel fibre described earlier has much less air fraction, no regularity, is single material all-silica, and the mode is largely determined by diffractive guidance even for the fundamental mode rather than an average step-index like effect. On the other hand, related diffractive coupling loss is a characteristic of regular photonic crystal fibres with the smallest microbends. Therefore, a comparison of both temperature and strain effects of the fundamental mode within a single material Fresnel fibre and a single-material photonic crystal fibre will further illustrate just how much flexibility in design exists by controlling structure alone.

From Figure 11, the transmission spectrum of a 4ring photonic crystal fiber with a fibre grating has two reflection bands corresponding with the two modes it is designed to support, one at longer wavelengths (1535.2 nm) for the fundamental mode, and the other $\sim 4 \mathrm{~nm}$ at shorter wavelengths for the higher-order mode. Figure 12 shows the obtained temperature dependencies for each mode-both grating transmission bands are found to have a similar linear variation with temperature. These results are in accordance with the material properties of the fiber, since the thermal expansion coefficient, $\alpha_{\mathrm{SiO} 2}$, of the fiber $\left(\alpha_{\mathrm{SiO} 2} \sim 0.55 \times 10^{-6}\right.$ for silica) is an order of magnitude smaller than the thermooptic coefficient-for germanium-doped silica core fiber this is $\left(1 / n_{\text {eff }}\right)\left(\partial n_{\text {eff }} / \partial T\right) \sim 8.6 \times 10^{-6}$, which serves as a reference value [81]. From the grating response as a function of temperature, the fundamental mode wavelength dependence is $\partial \lambda_{B 1} / \partial T=19.72 \mathrm{pm} /{ }^{\circ} \mathrm{C}$, and for $1535.2 \mathrm{~nm}$ of $\partial \lambda_{B 2} / \partial T=$ $20.14 \mathrm{pm} /{ }^{\circ} \mathrm{C}$. These correspond to $\left(1 / n_{\text {eff1 }}\right)\left(\partial n_{\text {eff1 }} / \partial T\right)=$ $12.9 \times 10^{-6}{ }^{\circ} \mathrm{C}^{-1}$, and $\left(1 / n_{\text {eff2 }}\right)\left(\partial n_{\text {eff2 }} / \partial T\right)=12.8 \times 10^{-6}{ }^{\circ} \mathrm{C}^{-1}$ which are nearly double that of pure silica although similar 


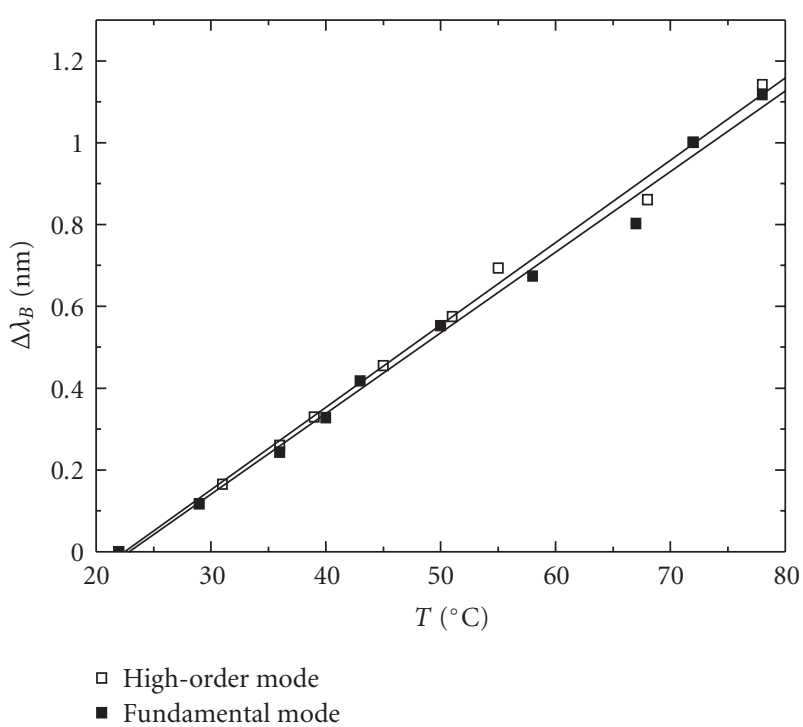

Figure 12: Temperature dependence of the two modes of the fibre described in Figure 11 [80].

to doped optical fibres. This larger value is due to the $\mathrm{Er}^{3+}$ doped aluminosilicate core, and it is not surprising that the higher-order mode, which sees more silica, has a slightly lower value. Therefore, the composite system behavior is expected on the basis of constituent component materials.

5.2. Strain Dependence. In contrast to the temperature dependencies, there is a difference between the fundamental mode and the higher-order mode of the previous $\mathrm{Er}^{3+}$ doped aluminosilicate photonic crystal fibre. The observed shift with applied strain in the Bragg wavelength corresponding to the fundamental mode (Figure 13) has a linear behavior $\left(\partial \lambda_{B 1} / \partial \varepsilon=1.2 \mathrm{pm} / \mu \varepsilon\right)$. However, the shorter Bragg wavelength, corresponding to the higher-order mode, has nonlinear behavior described by a quadratic dependence. Unlike conventional fibers, the higher-order leaky modes (in particular) of a photonic crystal fiber are sensitive to changes in stress between the holes and both the hole size and shape as well as the ratio of the hole diameter over the hole pitch, $d / \Lambda$ [82]. The compression of the first ring, in part arising as the solid core resists the compressive force, leads to an increase in $d / \Lambda$ with positive strain (applied tension). This problem is a well-known one that also contributes to hole deformation of the first ring during fiber fabrication if not addressed [83]. By increasing $d / \Lambda$, the modal confinement loss decreases, leading to improved confinement of the mode. This alters the fractions of power, $\eta$, in the center of the core and in both the silica ring and cladding. Since the effective index is dependent on this fraction, there is an additional shift in the Bragg wavelength $\left(\lambda_{B}=2 n_{\text {eff }} d\right)$ that deviates the curve from linearity. Given that the changes are based on circular confinement and therefore mode area, to first approximation a quadratic dependence $\left(\lambda \propto a \varepsilon+b \varepsilon^{2}\right)$ is expected and observed [80]. The strain-optic coefficient, therefore, reduces to $\partial \lambda_{B 1} / \partial \varepsilon=a+2 b \varepsilon$. Further, the

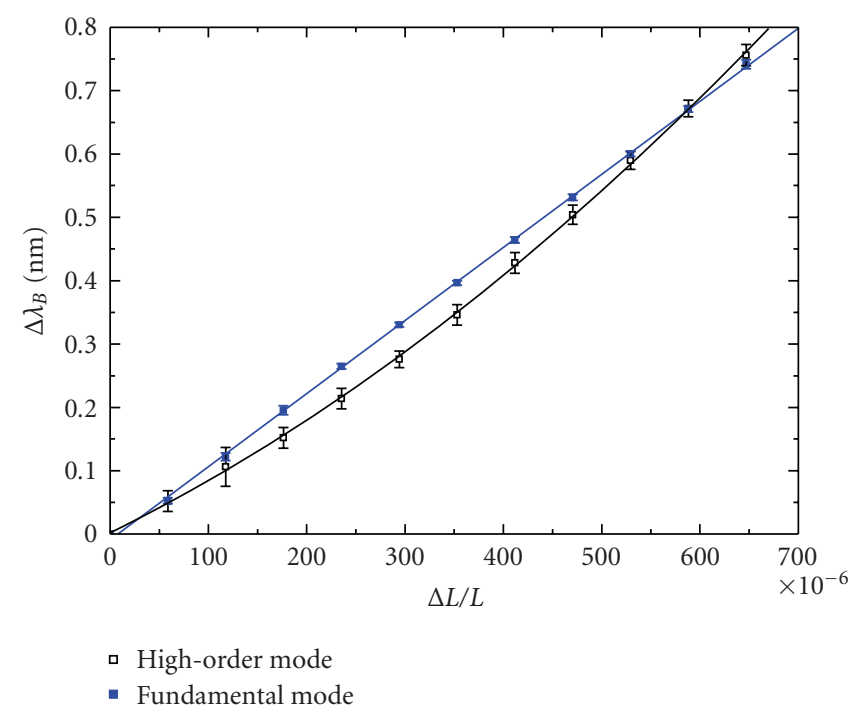

FIGURE 13: Wavelength shift versus applied longitudinal strain on the $\mathrm{Er}^{3+}$ doped, aluminosilicate dual mode photonic crystal fibre. A quadratic dependence is observed for the higher-order mode [80, 84].

grating strength is also dependent on this fraction $[\kappa=$ $\tan h^{2}(\pi \Delta \nu L \eta / \lambda)$ and we expect to observe a change in grating strength as a function of strain. The air structure itself becomes integral to defining the material properties and in this context is indistinguishable from a composite material. Therefore, the whole medium can be considered a unique super-structured material (SSM) with properties that can be tailored by tailoring the refractive index in a way analogous to tailoring the atomic distribution of constituents and their fractions in composite systems.

In contrast to this fibre, and despite the large interaction of the sensitive fundamental diffractive mode with the structure given its reduced confinement and the absence of an insulating step index core, the Fresnel fibre has a linear dependence with strain, as shown in Figure $14[58,84]$. This is explained by the very low air fraction and the irregular arrangement of holes that prevent a sponge-like structure susceptible to compressive effects that sufficiently affect the mode overlap with the holes.

The most obvious application of this work is to enable a simple distinction between strain and temperature from the perspective of controlling strain instead of temperature. The ability to remove, or unravel, the strain contribution is a key problem in fibre sensing with gratings generally and structured optical fibres offer a unique pathway to resolving this.

\section{Refractive Index Measurements Using Photonic Crystal Fibres}

As a consequence of the high core-cladding index contrast, the contribution to mode propagation from, for example, a periodic arrangement of holes is negligible when the wavelength of light is larger than the bridge thickness 


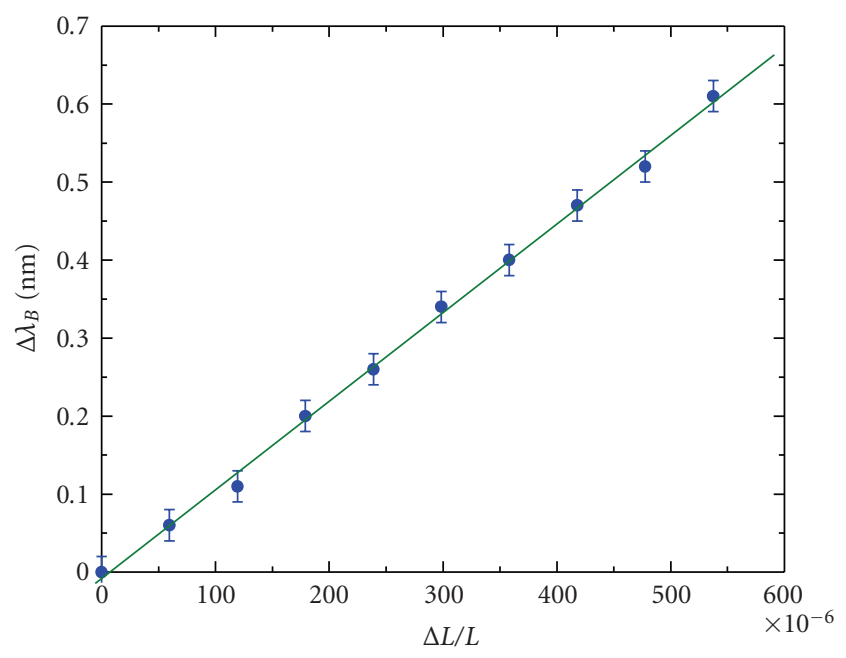

FIGURE 14: Wavelength shift versus applied longitudinal strain on the Fresnel fibre. No higher-order mode is observed in this fibre [58].

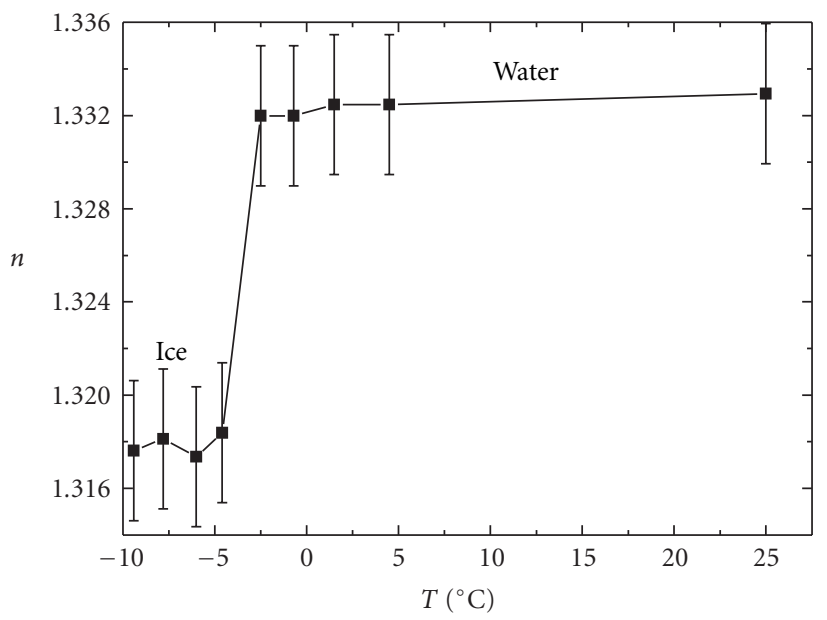

FIGURE 15: Refractive index measurement of the water and ice $1 \mathrm{~h}$ before, during, and after freezing [29].

between the holes but smaller than the core diameter. On the other hand, for less confined modes or when the wavelength of light is commensurate or smaller than the interstitial hole spacing, as well as the core size itself, a periodic lattice provides phase conditions that allow coherent scattering of light and therefore diffractive confinement [85]. This resonant phenomenon has been observed in bending loss tests where light leaks out from the core and is launched into the cladding, generating a short wavelength cutoff in the fibre transmission band [28]. Given that this property is unique to periodically structured optical fibres, it can be exploited in a unique fashion to measure the properties of what is placed into the holes.

The short wavelength cutoff is sensitive to the perturbations applied to the fibre as well as the index of the material within the hole. In fact, such regular coupling of light between the interstitial regions of a regular lattice structured fibre plays a key role in high bend loss characteristic of such fibres [56]. The best way to prevent this is therefore to remove the crystalline regularity of this lattice thereby spoiling the coupling - zero bend loss has been demonstrated using a chirped Fresnel fibre (or fractal fibre) [56]. Such optical fibres were also ideal as the basis for tapered structured fibres for efficient metal free near field microscopy [86], an important tool for diagnostic analysis which removes the problems of plasmon coupling in metal coated tips.

A $1 D$ variation of this diffractive scattering problem was observed within air-clad optical fibres. It arose from the regular corrugations of the surrounding air hole ring [87]. These particular fibres are especially important for astrophotonics and imaging applications because they can have extraordinarily high numerical apertures as a result of approaching the idealised air ring fibre. Recently, careful consideration to the effect on focal ratio degradation (FRD) was reported [88] — whilst no significant impact from diffractive scattering was noted, scattering generally off the corrugated surface was observed to give rise to degradation in FRD.

For the crystal lattice fibre, the corrugations associated with densification gratings produce the necessary coupling to the lattice through scattering [29]. The condition for the wavelength dependence of this scattering will be sensitive to what is in the air channels - in effect a diagnostic tool for measuring refractive index is possible. The refractive index of ice was measured this way, confirming how a simple Bragg condition suffices to describe the processes at short wavelengths. Therefore, the refractive index of the material within the holes, $n_{h}$, is given by [29]

$$
n_{h}=\frac{n_{\text {core }} \cos \left[\sin ^{-1}(\lambda m / 2 n \Lambda)\right]-x_{\mathrm{SiO}_{2}} n_{\mathrm{SiO}_{2}}}{x_{h}}
$$

where $n_{\text {cladding }}=x_{h} n_{h}+x_{\mathrm{SiO} 2} n_{\mathrm{SiO} 2}, x_{\mathrm{SiO} 2}$ and $x_{h}$ are the fractions of silica and holes that make up the cladding, $m$ is the grating order, $\Lambda$ is the lattice pitch, and $n_{\mathrm{SiO}_{2}}$ is the index of $\mathrm{SiO}_{2}$. Figure 15 shows the calculated refractive index of water determined from the transmission band edge shift in the visible to longer wavelengths as a function of temperature. Freezing is observed to occur at a lower temperature than zero, $\sim-3{ }^{\circ} \mathrm{C}$, consistent with very high pressures induced within the microchannel. To lower the m.p. of ice by such an amount requires a local pressure $>30 \mathrm{Mpa}$ [89], giving an indication of the high effective pressures that can be generated within micro-and nanocapillaries of ice $1 \mathrm{~h}$, which has $\sim 9 \%$ volume increase from the liquid state. This has important implications for micro-and nanofluidics. The calculated refractive index for the frozen ice is in agreement within error with that of ice $1 \mathrm{~h}$ as expected.

It is also possible to determine the refractive index of a material within the holes by monitoring the effective temperature dependence of an inscribed grating - the index, and its temperature dependence, can be extracted from the effective thermo-optic coefficient ( or $d n / d T$ ) of the fibre and its effective overlap derived form reference samples used to calibrate the sensor. Such a method was used to determine the thermo-optic coefficient of perfluoroheptane, which was not available in the literature [22]. Figure 16 shows the measurement of effective index change as a function of 


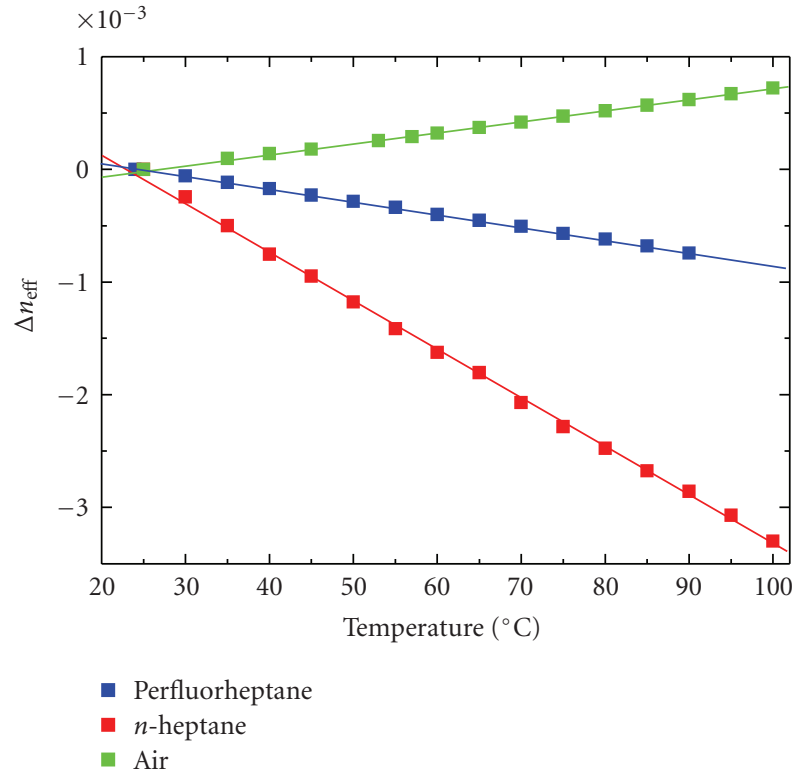

FIGURE 16: Effective index determined from shift in Bragg wavelength as a function of temperature. Heptane is used a reference to determine the actual $d n / d T$ of perfluoroheptane as [22].

temperature for both the unknown perfouroheptane and the known heptane. Substituting $\mathrm{H}$ for $\mathrm{F}$ in heptane significantly reduces the negative thermo-optic coefficient of the organic molecular system.

\section{Other Examples Based on Filling Structured Optical Fibres}

Filling of structured optical fibres has already produced simple but interesting all-fibre results. These include the demonstration of substantially enhanced stimulated Raman scattering (SRS) within a photonic bandgap fibre using hydrogen gas [90]. Simply using structured fibres as a cell for direct optical detection of gases such as methane and acetylene has also been demonstrated [91, 92]. For these latter applications it is clear that side access of the fibre, either by etching, laser ablation [93-96], or ion beam processing [97] will become important. Straightforward functional devices using liquid crystals have also been demonstrated, some of which have already been described earlier. A critical factor in taking this relatively straightforward approach to device fabrication to another level that truly differentiates structured optical fibres has been the development of selective filling, initially by filling the core only of a diffractive Fresnel fibre [98]. The techniques for allowing this to be done laid the foundation for the first true material engineering of an optical fibre by superposing the properties of three different materials without mixing them [24], a critical step to demonstrating the feasibility of lab-in-a-fibre technology [25] where multiple functionality, perhaps using all the above examples in the one fibre along with gratings and various other components, becomes possible. Optical localisation at the interface may play a critical role in condensing the feature sizes possible within a complex lab-in-a-fibre system, including the incorporation of semiconductors, polymers, and soft glasses to help raise the index contrast. A schematic of the potential lab-in-a fibre system is shown in Figure 17.

\section{Conclusions}

The purpose of the paper was to highlight some of the properties of the new generation optical fibres and gratings that are coming to fruition whilst also showing that conventional fibre technologies retain huge potential for further development. Starting with step index structured fibres [20], through to proposed Bragg bandgap fibres [99], demonstrated in structured form [100], to the step-index crystal lattices developed in a similar direction [101] and the subsequent bandgap fiber experimental results [102] based on theoretical designs [103], with later experimental results more convincing [104], to the quasicrystal, zone plate Fresnel fibres $[30-34,84,105]$, structured fibres have the potential for extending optical fibre capability well beyond conventional technologies. Nevertheless, the literature database for both conventional and structured optical fibres is now so large and rapidly growing that it is impossible to do fair justice to all examples developed. The select few chosen here, largely air-structured fibres, are aligned with the perspective of exploiting these new trends.

Material science is clearly an increasingly important determinant for application specific devices and systems. Given the wide exploration space into new materials, especially soft glasses and polymers, the paper has focussed specifically on silica-related technologies as these remain by far the most mature and practical with regards to applications within the sensing industries. However, in some areas these other materials (ranging from disposable and soluble cellulose fibres for biodiagnostics [106] through arbitrary structured fibre cross-section using polymer extrusion [107] to soft glasses for infrared operation $[108,109]$ through to semiconductor optical fibres [110] including new silicon core silica-based fibres [111]) will have an important role to play. In this respect, it is worth digressing and commenting briefly on the next material system heralded for fibre Bragg gratings sensor work: polymer fibres.

Grating writing in other materials is sometimes, in relation to the work done in silica, contentious. Recent demonstrations of gratings in polymer structured fibres, for example, are shown in reflection only because they have been virtually undetectable in transmission $[112,113]$, similar to initial work in regenerated gratings [64]. Further, in addition to the very large losses associated with these structured polymer fibres, mostly made of polymethylmethacrylate (PMMA), there is evidence that the gratings written into them are made up of periodic UV oxidation of the polymer in air along the fibre-in addition to observable coloration with longer exposures, when nitrogen was used to displace air no gratings were able to be inscribed [113]. In contrast, however, some interesting results in solid polymer fibre with a special photosensitiser such as trans-4-stilbenemethanol [112-115] do promise potential applications in biomedicine 


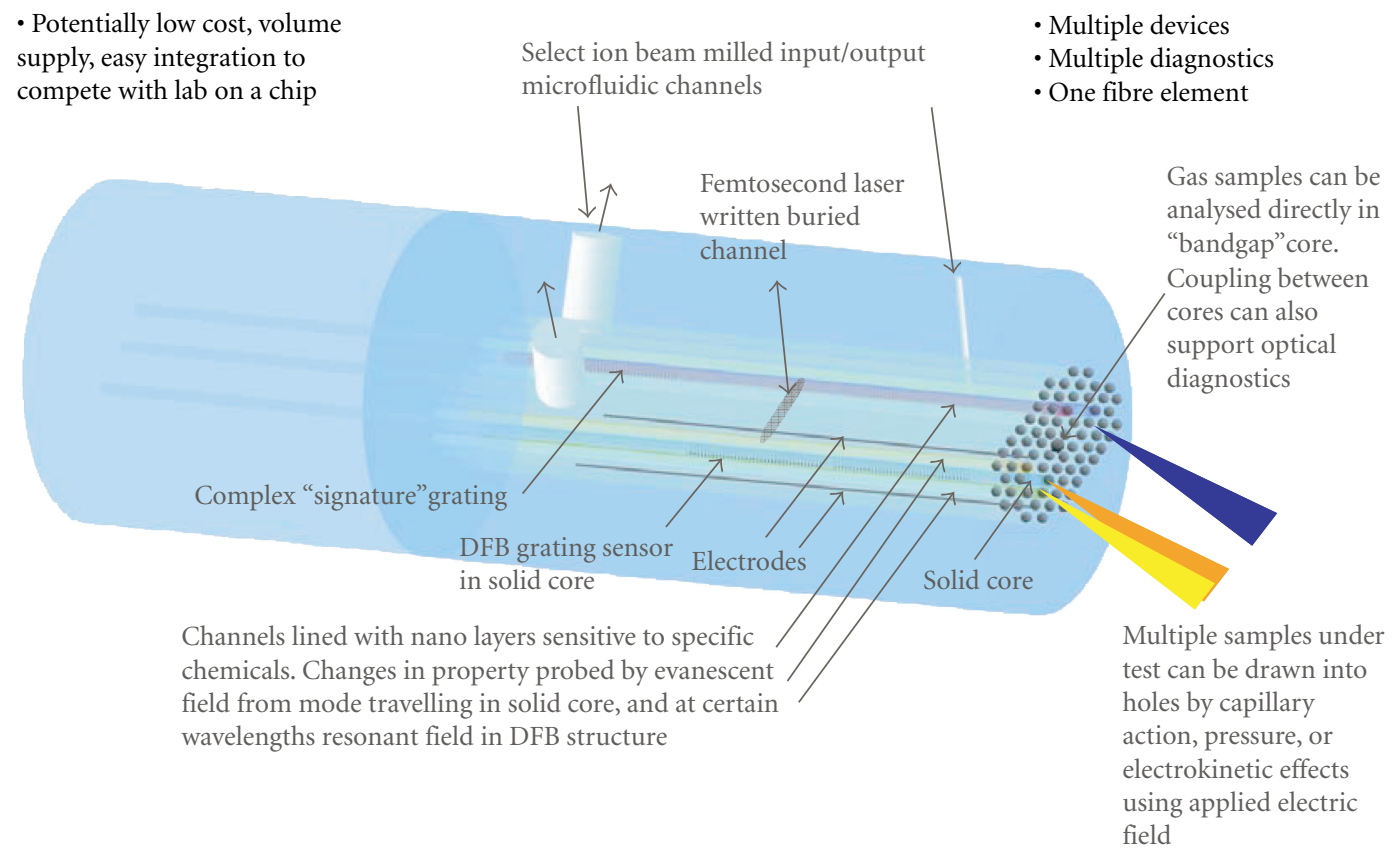

FIGURE 17: Schematic of multiple functionality within a possible future lab in a fibre.

where some organic compatibility is required. (The issue of biocompatibility is a complex one that needs full peer review within the medical community, particularly given the increased range of materials, and dopants, being proposed for biomedical applications that often involve invasive procedures within organic tissue.) This is significant progress from the first results in polymer fibre [116] where high propagation losses made implementation impossible. It is clear, nonetheless, to anyone who has worked in this area that there still remains enormous materials work to advance polymer fibre gratings to applications. A key problem that has to be investigated is deformation and the impact on long-term performance-this is particularly problematic for structured polymer optical fibres which have been proposed by the author and others for LAN networks. On the other hand, this detrimental aspect of polymer structured optical fibres has been exploited to make effective point sensors for the first force measurements within orthodontic applications [3, 117]. The ability to incorporate polymer (or other materials including silicon [111]) materials within and around silica structured fibres, including photosensitive systems, offers a simple interim solution for biocompatible applications as well as a viable access route to the large strain and thermo-optic coefficients of polymers (recall the use of organic fluids within structured silica fibre for such effects).

With regards to grating writing in soft glasses, this should see relatively straightforward success, since index changes should be easy to achieve using visible laser light to access the band edge and/or specific defect sites, although little work has yet been reported in fibre form. The question of long-term stability, however, will likely pose further material challenges.
The future of the silica fibre Bragg grating, therefore, as the lynch pin for optical fibre sensing looks bright. New writing technologies, such as femtosecond laser processing, continue to be explored-these enable writing in almost any material, for example, and the technologies have been covered in greater detail within other reviews $[52,61-63$, 76]. Meanwhile, a structured optical fibre potentially allows, using both air holes and filled glass systems, unprecedented custom tailoring of both a fibre and a gratings properties in terms of genuine composite material engineering. Despite all these new developments and promises, however, type I grating writing within conventional fibres continues to persist as an active starting point for new grating research. Regenerated gratings that operate in excess of $1200^{\circ} \mathrm{C}$ are a novel highlight. These "conventional" gratings are particularly attractive as they retain many important practical features of conventional fibres that the new generation of fibres has yet to fulfil: low splice losses, low propagation losses, no interior contamination with breaks, and general overall simplicity and compatibility with existing maintenance technology. Further, the grating writing process using CW $244 \mathrm{~nm}$ light remains the most commercially accepted and by far the most advanced, enabling any spectral filter design for numerous applications. On the other hand, new structured fibres offer alternative routes to solve many of the problems of existing conventional fibres. The decision of which technology to use will depend on whether the maturity of practical conventional fibre technology can be outweighed by new functionality that structured fibres offer for a specific application. The optimistic longer-term directions for sensing, epitomised by the potential of labin-a-fibre technology, look promising indeed. On the other hand, for many applications simplicity is central to a working 
design — a good example that also highlights the potential of structured fibre technology is a simple hydrostatic pressure sensor based on a grating written into a germanosilicate core fibre with two large side holes [118]. Simply breaking the symmetry of the fibre using holes breaks the isotropic response to hydrostatic pressure and allows induced grating birefringence to serve as direct, and effective, monitor of pressure. The sensitivity can obviously be enhanced with a passive or active resonant structure.

\section{Acknowledgments}

The author would like to acknowledge various students, staff, and colleagues. In particular the following: Nathaniel Groothoff, Kevin Cook, Michael Stevenson, Jacob Fenton, Mattias Aslund from IPL; Cicero Martelli now at the Mechanical Engineering Department, PUC, Rio Brazil; Hypolito Kalinowski and Alexandre Pohl from the Federal University of Technology, Curitiba, Parana, Brazil; John Holdsworth from the Mathematical and Physical Sciences School, Newcastle University, Newcastle, NSW, Australia; Somnath Bandyopadhyay from the Central Glass and Ceramic Research Institute (CGCRI), Kolkata, India; Henrick Sorenson, formerly Centre COM, DTU, now at Koheras A/S, Denmark, Hans Deyerl formerly Centre COM, DTU, now with Chemnitz university of Technology, Germany, Martin Kristensen formerly Centre COM, DTU, now with iNANO, Arhus university, Denmark and other colleagues from Research Centre COM (now DTU Fotonik, Department of Photonic Engineering, Denmark) and many others. Funding for this work came from the Australian Research Council through its Discovery Project scheme and the Department of Education, Science and Training (DEST), Australia through its International Science Linkages programs.

\section{References}

[1] BCC, "Fiber Optic Sensors," (Report code: 1AS002D), 2009, http://www.photonics.com.

[2] H. J. Kalinowski, I. Abeb, J. A. Simõesc, and A. Ramosc, "Application of fibre Bragg grating sensors in biomechanics," in Trends in Photonics, J. Canning, Ed., chapter 10, Research Signpost, Kerala, India, 2008.

[3] M. S. Milczewski, J. C. Cardozo da Silva, L. Carvalho, J. Canning, and H. J. Kalinowski, "Optical fiber sensors in dentistry," in Trends in Photonics, J. Canning, Ed., chapter 11, Research Signpost, Kerala, India, 2008.

[4] F. I. Baldini and A. G. Mignani, "Biomedical fibre optic sensors," in Handbook of Optical Fibre Sensing Technology, J. M. López-Higuera, Ed., Wiley Interscience, Chichester, UK, 2002.

[5] C. Martelli, J. Canning, J. R. Reimers, et al., "Evanescentfield spectroscopy using structured optical fibers: detection of charge-transfer at the porphyrin-silica interface," Journal of the American Chemical Society, vol. 131, no. 8, pp. 29252933, 2009.

[6] I. Latka, W. Ecke, B. Hoefer, T. Frangen, R. Willsch, and A. Reutlinger, "Micro bending beam based optical fiber grating sensors for physical and chemical measurands," in
Proceedings of the 17th International Conference on Optical Fibre Sensors, vol. 5855 of Proceedings of SPIE, pp. 94-97, Bruges, Belgium, May 2005.

[7] W. Du, X. M. Tao, H. Y. Tam, and C. L. Choy, "Fundamentals and applications of optical fiber Bragg grating sensors to textile structural composites," Composite Structures, vol. 42, no. 3, pp. 217-229, 1998.

[8] R. Willsch, W. Ecke, and H. Bartelt, "Optical fiber grating sensor networks and their application in electric power facilities, aerospace and geotechnical engineering," in Proceedings of the 15th Optical Fiber Sensors Conference Technical Digest (OFS '02), pp. 49-54, Portland, Ore, USA, May 2002.

[9] B. Lee, "Review of the present status of optical fiber sensors," Optical Fiber Technology, vol. 9, no. 2, pp. 57-79, 2003.

[10] I. Latka, W. Ecke, B. Höfer, C. Chojetzki, and A. Reutlinger, "Fiber optic sensors for the monitoring of cryogenic spacecraft tank structures," in Photonics North 2004: Photonic Applications in Telecommunications, Sensors, vol. 5579 of Proceedings of SPIE, pp. 195-204, Ottawa, Canada, November 2004.

[11] W. Ecke, K. Schroeder, M. Kautz, et al., "On-line characterization of impacts on electrical train current collectors using integrated optical fiber grating sensor network," in Smart Structures and Materials 2005: Smart Sensor Technology and Measurement Systems, vol. 5758 of Proceedings of SPIE, pp. 114-123, San Diego, Calif, USA, March 2005.

[12] K. Schroeder, W. Ecke, J. Apitz, E. Lembke, and G. Lenschow, "A fibre Bragg grating sensor system monitors operational load in a wind turbine rotor blade," Measurement Science and Technology, vol. 17, no. 5, pp. 1167-1172, 2006.

[13] J. C. Cardozo da Silva, C. Martelli, H. J. Kalinowski, E. Penner, J. Canning, and N. Groothoff, "Dynamic analysis and temperature measurements of concrete cantilever beam using fibre Bragg gratings," Optics and Lasers in Engineering, vol. 45, no. 1, pp. 88-92, 2007.

[14] A. C. L. Wong, P. A. Childs, R. Berndt, T. Macken, G.D. Peng, and N. Gowripalan, "Simultaneous measurement of shrinkage and temperature of reactive powder concrete at early-age using fibre Bragg grating sensors," Cement and Concrete Composites, vol. 29, no. 6, pp. 490-497, 2007.

[15] H. Soejima, T. Ogisu, H. Yoneda, Y. Okabe, N. Takeda, and Y. Koshioka, "Demonstration of detectability of SHM system with FBG/PZT hybrid system in composite wing box structure," in Sensors and Smart Structures Technologies for Civil, Mechanical, and Aerospace Systems, vol. 6932 of Proceedings of SPIE, San Diego, Calif, USA, March 2008.

[16] V. G. M. Annamdas, Y. Yang, and H. Liu, "Current development in fiber Bragg grating sensors and their applications," in Sensors and Smart Structures Technologies for Civil, Mechanical, and Aerospace Systems, vol. 6932 of Proceedings of SPIE, San Diego, Calif, USA, March 2008.

[17] M. Majumder, T. K. Gangopadhyay, A. K. Chakraborty, K. Dasgupta, and D. K. Bhattacharya, "Fibre Bragg gratings in structural health monitoring-present status and applications," Sensors and Actuators A, vol. 147, no. 1, pp. 150-164, 2008.

[18] J. A. Epaarachchi, J. Canning, and M. Stevenson, "Investigation of the response of embedded Near Infrared FBG $(830 \mathrm{~nm})$ sensors in glass fibre composites," in Proceedings of 19th International Conference on Optical Fibre Sensors, vol. 7004 of Proceedings SPIE, 2008.

[19] A. C. L. Wong, P. A. Childs, and G.-D. Peng, "Spectrally coded multiplexing techniques in fibre-optic sensor systems," 
in Trends in Photonics, J. Canning, Ed., chapter 8, Research Signpost, Kerala, India, 2008.

[20] P. Kaiser and H. W. Astle, "Low-loss single-material fibers made from pure fused silica," Bell System Technical Journal, vol. 53, no. 6, pp. 1021-1039, 1974.

[21] K. Digweed-Lyytikainen, C. A. de Francisco, D. Spadoti, et al., "Photonic crystal optical fibers for dispersion compensation and Raman amplification: design and experiment," Microwave and Optical Technology Letters, vol. 49, no. 4, pp. 872-874, 2007.

[22] H. R. Sørenson, J. Canning, J. Lægsgaard, K. Hansen, and P. Varming, "Control of the wavelength dependent thermooptic coefficients in structured fibres," Optics Express, vol. 14, no. 14, pp. 6428-6433, 2006.

[23] N. Mothe, D. Pagnoux, H. Phan, et al., "Thermal wavelength stabilization of Bragg gratings photowritten in hole-filled microstructured optical fibers," Optics Express, vol. 16, no. 23, pp. 19018-19033, 2008.

[24] J. Canning, M. Stevenson, T. K. Yip, S. K. Lim, and C. Martelli, "White light sources based on multiple precision selective micro-filling of structured optical waveguides," Optics Express, vol. 16, no. 20, pp. 15700-15708, 2008.

[25] J. Canning, "New trends in structured optical fibres for telecommunications and sensing," in Proceedings of the 5th International Conference on Optical Communications and Networks and the 2nd International Symposium on Advances and Trends in Fiber Optics and Applications (ICOCN/ATFO '06), Chengdu, China, 2006.

[26] C. Martelli, J. Canning, and B. Ashton, "Add drop gas reference cell with acetylene," in Proceedings of the Optical Fiber Sensors Conference (OFS '06), Cancun, Mexico, 2006.

[27] A. Bjarklev, J. Broeng, and A. S. Bjarklev, Photonic Crystal Fibres, Kluwer Academic Publishers, Dordrecht, The Netherlands, 2003.

[28] D. Kácik, I. Turek, I. Martincek, J. Canning, and K. Lyytikäinen, "The role of diffraction in determining the short wavelength losses edge of photonic crystal fibres," in Proceedings of the Australian Conference on Optical Fibre Technology (ACOFT '05), Sydney, Australia, 2005.

[29] C. Martelli, J. Canning, M. Kristensen, and N. Groothoff, "Refractive index measurement within a photonic crystal fibre based on short wavelength diffraction," Sensors, vol. 7, no. 11, pp. 2492-2498, 2007.

[30] J. Canning, "Fresnel optics inside optical fibres," in Photonics Research Developments, Nova Science Publishers, Hauppauge, NY, USA, 2009.

[31] J. Canning, E. Buckley, and K. Lyytikäinen, "Propagation in air by field superposition of scattered light within a Fresnel fibre," Optics Letters, vol. 28, no. 4, pp. 2330-2332, 2003.

[32] J. Canning, E. Buckley, and K. Lyytikäinen, "Multiple source generation using air-structured optical waveguides for optical field shaping and transformation within and beyond the waveguide," Optics Express, vol. 11, no. 4, pp. 347-358, 2003.

[33] J. Canning, E. Buckley, and K. Lyytikäinen, "All-fibre phaseaperture zone plate fresnel lenses," Electronics Letters, vol. 39, no. 3, pp. 311-312, 2003.

[34] C. Martelli and J. Canning, "Fresnel fibres with core-defects for optical sensing," in Proceedings of Optical Fiber Sensors Conference (OFS '06), Cancun Mexico, 2007, postdeadline paper, reproduced in C. Martelli, $\mathrm{PhD}$ dissemination, University of Sydney, 2007.

[35] V. R. Almeida, Q. Xu, C. A. Barrios, and M. Lipson, "Guiding and confining light in void nanostructure," in Optics Letters, vol. 29, no. 11, pp. 1209-1211, June 2004.
[36] B. Schmidt, V. Almeida, C. Manolatou, S. Preble, and M. Lipson, "Nanocavity in a silicon waveguide for ultrasensitive nanoparticle detection," Applied Physics Letters, vol. 85, no. 21, pp. 4854-4856, 2004.

[37] N. Skivesen, A. Têtu, M. Kristensen, J. Kjems, L. H. Frandsen, and P. I. Borel, "Photonic-crystal waveguide biosensor," Optics Express, vol. 15, no. 6, pp. 3169-3176, 2007.

[38] N. Skivesen, J. Canning, M. Kristensen, C. Martelli, A. Tetu, and L. H. Frandsen, "Photonic crystal waveguidebased biosensor," in Proceedings of Optical Fiber Communication/National Fiber Optic Engineers Conference (OFC/NFOEC '08), pp. 1-3, San Diego, Calif, USA, February 2008.

[39] G. S. Wiederhecker, C. M. B. Cordeiro, F. Couny, et al., "Field enhancement within an optical fibre with a subwavelength air core," Nature Photonics, vol. 1, no. 2, pp. 115-118, 2007.

[40] C. M. Rollinson, S. M. Orbons, S. T. Huntington, et al., "Metal-free scanning optical microscopy with a fractal fiber probe," Optics Express, vol. 17, no. 3, pp. 1772-1780, 2009.

[41] C. Martelli, J. Canning, and K. Lyytikäinen, "Water core Fresnel fibre," Optics Express, vol. 13, no. 10, pp. 3890-3895, 2005.

[42] S. Donati, Electro-Optical Instrumentation: Sensing and Measuring with Lasers, Prentice-Hall, Englewood Cliffs, NJ, USA, 2004.

[43] U. C. Paek and C. R. Kurkjian, "Calculation of cooling rate and induced stresses in drawing of optical fibers," Journal of the American Ceramic Society, vol. 58, no. 7-8, pp. 330-335, 1975.

[44] G. W. Scherer and A. R. Cooper, "Thermal stresses in cladglass fibers," Journal of the American Ceramic Society, vol. 63, no. 5-6, pp. 346-347, 1980.

[45] Y. Park, T.-J. Ahn, Y. H. Kim, W.-T. Han, U.-C. Paek, and D. Y. Kim, "Measurement method for profiling the residual stress and the strain-optic coefficient of an optical fiber," Applied Optics, vol. 41, no. 1, pp. 21-26, 2002.

[46] B. H. Kim, Y. Park, D. Y. Kim, U. C. Paek, and W.-T. Han, "Observation and analysis of residual stress development resulting from $\mathrm{OH}$ impurity in optical fibers," Optics Letters, vol. 27, no. 10, pp. 806-808, 2002.

[47] O. V. Mazurin, M. V. Streltsina, and T. P. ShvaikoShvaikovskaya, Handbook of Glass Data Part A: Silica Glass and Binary Silicate Glasses, vol. 15 of Physical Sciences Data, Elsevier, Amsterdam, The Netherlands, 1983.

[48] J. Bland-Hawthorn, M. Englund, and G. Edvell, "New approach to atmospheric $\mathrm{OH}$ suppression using an aperiodic fibre Bragg grating," Optics Express, vol. 12, no. 24, pp. 59025909, 2004.

[49] B. Leconte, W.-X. Xie, M. Douay, et al., "Analysis of colorcenter-related contribution to Bragg grating formation in $\mathrm{Ge}: \mathrm{SiO}_{2}$ fiber based on a local Kramers-Kronig transformation of excess loss spectra," Applied Optics, vol. 36, no. 24, pp. 5923-5930, 1997.

[50] H. G. Limberger, P.-Y. Fonjallaz, R. P. Salathé, and F. Cochet, "Compaction- and photoelastic-induced index changes in fiber Bragg gratings," Applied Physics Letters, vol. 68, no. 22, pp. 3069-3071, 1996.

[51] N. H. Ky, H. G. Limberger, R. P. Salathé, F. Cochet, and L. Dong, "UV-irradiation induced stress and index changes during the growth of type-I and type-IIA fiber gratings," Optics Communications, vol. 225, no. 4-6, pp. 313-318, 2003.

[52] J. Canning, "Fibre gratings and devices for sensors and laser," Laser and Photonics Reviews, vol. 2, no. 4, pp. 275-289, 2008. 
[53] P. K. Bachmann, W. Hermann, H. Wehr, and D. U. Wiechert, "Stress in optical waveguides. 1: preforms," Applied Optics, vol. 25, no. 7, pp. 1093-1098, 1986.

[54] P. K. Bachmann, W. Hermann, H. Wehr, and D. U. Wiechert, "Stress in optical waveguides. 2: fibers," Applied Optics, vol. 26, no. 7, pp. 1175-1182, 1987.

[55] J. Canning, M. Stevenson, S. Bandyopadhyay, and K. Cook, "Extreme silica optical fibre gratings," Sensors, vol. 8, no. 10, pp. 6448-6452, 2008.

[56] C. Martelli, J. Canning, B. Gibson, and S. Huntington, "Bend loss in structured optical fibres," Optics Express, vol. 15, no. 26, pp. 17639-17644, 2007.

[57] N. Groothoff, J. Canning, E. Buckley, K. Lyttikainen, and J. Zagari, "Bragg gratings in air-silica structured fibers," Optics Letters, vol. 28, no. 4, pp. 233-235, 2003.

[58] N. Groothoff, C. Martelli, J. Canning, and K. Lyytikäinen, "Fibre Bragg grating in Fresnel fibre with temperature and strain characterisation," in Proceedings of Australian Conference on Optical Fibre Technology (ACOFT'05), Sydney, Australia, 2005.

[59] J. Albert, B. Malo, F. Bilodeau, et al., "Photosensitivity in Gedoped silica optical waveguides and fibers with 193-nm light from an ArF excimer laser," Optics Letters, vol. 19, no. 6, pp. 387-389, 1994.

[60] J. Canning, H. G. Inglis, M. G. Sceats, and P. Hill, “Transient and permanent gratings in phosphosilicate optical fibers produced by the flash condensation technique," Optics Letters, vol. 20, p. 2189, 1995.

[61] J. Canning, "Gratings and grating devices in structured fibres using 193nm from an ArF laser," in Proceedings of the OSA Topical Meeting: Bragg Gratings, Photosensitivity and Poling (BGPP '07), Quebec City, Canada, 2007, (invited).

[62] J. Canning, Ed., Proceedings of the 1st International Workshop on Multiphoton Processes in Glass and Glassy Materials, Darlington Centre, University of Sydney, Sydney, Australia.

[63] S. J. Mihailov, D. Grobnic, C. W. Smelser, P. Lu, R. B. Walker, and H. Ding, "Bragg grating inscription using femtosecond laser sources," in Trends in Photonics, J. Canning, Ed., chapter 4, Research Signpost, Kerala, India, 2008.

[64] B. Zhang and M. Kahriziet, "High temperature resistance fiber Bragg grating temperature sensor fabrication," IEEE Sensors Journal, vol. 7, pp. 586-590, 2007.

[65] S. Bandyopadhyay, J. Canning, M. Stevenson, and K. Cook, "Ultra-high temperature regenerated gratings in boron codoped germanosilicate optical fibre using $193 \mathrm{~nm}$," Optics Letters, vol. 33, no. 16, pp. 1917-1919, 2008.

[66] M. Fokine, "Formation of thermally stable chemical composition gratings in optical fibers," Journal of the Optical Society of America B, vol. 19, no. 8, pp. 1759-1765, 2002.

[67] H. Dobb, K. Kalli, and D. J. Webb, "Temperature-insensitive long period grating sensors in photonic crystal fibre," Electronics Letters, vol. 40, no. 11, pp. 657-658, 2004.

[68] A. Michie, J. Canning, K. Lyytikäinen, M. Åslund, and J. Digweed, "Temperature independent highly birefringent photonic crystal fibre," Optics Express, vol. 12, no. 21, pp. 5160-5165, 2004.

[69] D.-H. Kim and J. U. Kang, "Sagnac loop interferometer based on polarization maintaining photonic crystal fiber with reduced temperature sensitivity," Optics Express, vol. 12, no. 19, pp. 4490-4495, 2004.

[70] C.-L. Zhao, X. Yang, C. Lu, W. Jin, and M. S. Demokan, "Temperature-insensitive interferometer using a highly birefringent photonic crystal fiber loop mirror," IEEE Photonics Technology Letters, vol. 16, no. 11, pp. 2535-2537, 2004.
[71] M. J. F. Digonnet, H. K. Kim, S. Blin, V. Dangui, and G. S. Kino, "Sensitivity and stability of an air-core fiber-optic gyroscope," in Optical Fiber Sensors, OSA Technical Digest (CD), Optical Society of America, 2006, paper ME1.

[72] A. Michie, J. Canning, I. Bassett, et al., "Spun elliptically birefringent photonic crystal fibre," Optics Express, vol. 15, no. 4, pp. 1811-1816, 2007.

[73] X. Dong, H. Y. Tam, and P. Shum, "Temperature-insensitive strain sensor with polarization-maintaining photonic crystal fiber based Sagnac interferometer," Applied Physics Letters, vol. 90, no. 15, Article ID 151113, 3 pages, 2007.

[74] D. Káčik, I. Turek, I. Martinček, J. Canning, N. A. Issa, and K. Lyytikäinen, "Intermodal interference in a photonic crystal fibre," Optics Express, vol. 12, no. 15, pp. 3465-3470, 2004.

[75] J. Villatoro, V. Finazzi, V. P. Minkovich, V. Pruneri, and G. Badenes, "Temperature-insensitive photonic crystal fiber interferometer for absolute strain sensing," Applied Physics Letters, vol. 91, no. 9, Article ID 091109, 2007.

[76] N. Groothoff, C. Martelli, and J. Canning, "A dual wavelength distributed feedback fibre laser," Journal of Applied Physics, vol. 103, Article ID 013101, 2008.

[77] J. Canning, N. Groothoff, K. Cook, et al., "Gratings in structured optical fibres," Laser Chemistry, vol. 2008, Article ID 239417, 2008.

[78] C. K. Kirkendall and A. Dandridge, "Overview of high performance fibre-optic sensing," Journal of Physics D, vol. 37, no. 18, pp. R197-R216, 2004.

[79] S. Foster, A. Tikhomirov, M. Englund, H. Inglis, G. Edvell, and M. Milnes, "A 16 channel fibre laser sensor array," in Proceedings of the Australian Conference on Optical Fibre Technology (ACOFT '06), pp. 40-42, Melbourne, Australia, 2006.

[80] C. Martelli, J. Canning, N. Groothoff, and K. Lyytikäinen, "Strain and temperature characterization of photonic crystal fiber Bragg gratings," Optics Letters, vol. 30, no. 14, pp. 1785$1787,2005$.

[81] M. Janos and J. Canning, "Permanent and transient resonances thermally induced in optical fibre Bragg gratings," Electronics Letters, vol. 31, no. 12, pp. 1007-1009, 1995.

[82] D. Ferrarini, L. Vincetti, M. Zoboli, A. Cucinotta, and S. Selleri, "Leakage properties of photonic crystal fibers," Optics Express, vol. 10, no. 23, pp. 1314-1319, 2002.

[83] K. Lyytikäinen, Control of complex structural geometry in optical fibre drawing, Ph.D. thesis, School of Physics and Interdisciplinary Photonics Laboratories, The University of Sydney, Sydney, Australia, 2004.

[84] C. Martelli, J. Canning, N. Groothoff, and K. Lyttikainen, "Bragg gratings in photonic crystal fibres: strain and temperature characterisation," in Proceedings of the 17th International Conference on Optical Fibre Sensors, M. Voet, R. Willsch, W. Ecke, J. Jones, and B. Culshaw, Eds., vol. 5855 of Proceedings of SPIE, Bellingham, Wash, USA, 2005.

[85] J. Canning, "Diffraction-free mode generation and propagation in optical waveguides," Optics Communications, vol. 207, no. 1-6, pp. 35-39, 2002.

[86] C. M. Rollinson, S. M. Orbons, S. T. Huntington, et al., "Metal-free scanning optical microscopy with a fractal fibre probe," Optics Express, vol. 17, no. 3, pp. 1772-1780, 2009.

[87] M. Åslund, J. Canning, S. D. Jackson, A. Teixeira, and K. Lyytikäinen, "Diffraction in air-clad fibres," Optics Express, vol. 13, no. 14, pp. 5227-5233, 2005.

[88] M. Åslund and J. Canning, "Air-clad fibres for astronomical instrumentation: focal-ratio degradation," Experimental Astronomy, vol. 24, no. 1-3, pp. 1-7, 2009. 
[89] V. F. Petrenko and R. W. Whitworth, Physics of Ice, Oxford University Press, New York, NY, USA, 2002.

[90] F. Benabid, J. C. Knight, G. Antonopoulos, and P. St. J. Russell, "Stimulated Raman scattering in hydrogen-filled hollow-core photonic crystal fiber," Science, vol. 298, no. 5592, pp. 399-402, 2002.

[91] T. Ritari, J. Tuominen, H. Ludvigsen, et al., "Gas sensing using air-guiding photonic bandgap fibers," Optics Express, vol. 12, no. 17, pp. 4080-4087, 2004.

[92] Y. L. Hoo, W. Jin, H. L. Ho, J. Ju, and D. N. Wang, "Gas diffusion measurement using hollow-core photonic bandgap fiber," Sensors and Actuators B, vol. 105, no. 2, pp. 183-186, 2005.

[93] J. Canning, E. Buckley, N. Groothoff, B. Luther-Davies, and J. Zagari, "UV laser cleaving of air-polymer structured fibre," Optics Communications, vol. 202, no. 1-3, pp. 139-143, 2002.

[94] J. Canning, E. Buckley, N. Groothoff, and S. Huntington, "Laser sculpting and shaping of air-polymer structured fibres," in Proceedings Australian Conference on Optical Fibre Technology (ACOFT '03), Melbourne, Australia, 2003.

[95] H. Lehmann, S. Brueckner, J. Kobelke, G. Schwotzer, K. Schuster, and R. Willsch, "Toward photonic crystal fiber based distributed chemosensors," in Proceedings of the 17th International Conference on Optical Fibre Sensors, vol. 5855 of Proceedings of SPIE, pp. 419-422, 2005.

[96] Y. Lai, K. Zhou, L. Zhang, and I. Bennion, "Fabrication of micro-channels in optical fibers using femtosecond laser pulses and selective chemical etching," Optics Letters, vol. 31, p. 2559, 2006.

[97] C. Martelli, P. Olivero, J. Canning, N. Groothoff, B. Gibson, and S. Huntington, "Micromachining structured optical fibres using focussed ion beam (FIB) milling," Optics Letters, vol. 32, no. 12, pp. 1575-1577, 2007.

[98] C. Martelli, J. Canning, and K. Lyytikainen, "Water core Fresnel fibre," Optics Express, vol. 13, no. 10, pp. 3890-3895, 2005.

[99] P. Yeh, A. Yariv, and E. Maron, "Theory of Bragg fiber," Journal of the Optical Society of America, vol. 68, no. 9, pp. 1196-1201, 1978.

[100] G. Vienne, Y. Xu, C. Jakobsen, et al., "Ultra-large bandwidth hollow-core guiding in all-silica Bragg fibers with nanosupports," Optics Express, vol. 12, no. 15, pp. 3500-3508, 2004.

[101] J. Knight, T. Birks, P. St. J. Russell, and D. M. Atkins, "All-silica singlemode optical fiber with photonic crystal cladding," Optics Letters, vol. 21, no. 19, pp. 1547-1549, 1996.

[102] J. C. Knight, J. Broeng, T. A. Birks, and P. St. J. Russell, "Photonic band gap guidance in optical fibers," Science, vol. 282, no. 5393, pp. 1476-1478, 1998.

[103] J. Broeng, S. E. Barkou, and A. Bjarklev, "Waveguiding by the photonic bandgap effect," in Proceedings of the Topical Meeting on Electromagnetic Optics, pp. 67-68, EOS, hyeres, France, 1998.

[104] N. Venkataramn, M. T. Gallagher, C. M. Smith, et al., "Low loss $(13 \mathrm{~dB} / \mathrm{km})$ air core photonic bandgap fibre," in Proceedings of the European Conference on Optical Communications (ECOC '02), Copenhagen, Denmark, 2002, PD1.1.

[105] C. Martelli and J. Canning, "Fresnel fibres with omnidirectional zone cross-sections," Optics Express, vol. 15, no. 7, pp. 4281-4286, 2007.

[106] A. Dupuis, N. Guo, Y. Gao, et al., "Prospective for biodegradable microstructured optical fibers," Optics Letters, vol. 32, no. 2, pp. 109-111, 2007.
[107] M. Mignanelli, K. Wani, J. Ballato, S. Foulger, and P. Brown, "Polymer microstructured fibers by one-step extrusion," Optics Express, vol. 15, no. 10, pp. 6183-6189, 2007.

[108] J. S. Sanghera and I. D. Aggarwal, Eds., Infrared Fiber Optics, CRC Press, Boca Raton, Fla, USA, 1998.

[109] H. Ebendorff-Heidepriem and T. M. Monro, "Extrusion of complex preforms for microstructured optical fibers," Optics Express, vol. 15, no. 23, pp. 15086-15092, 2007.

[110] S. D. Hart, G. R. Maskaly, B. Temelkuran, P. H. Prideaux, J. D. Joannopoulos, and Y. Fink, "External reflection from omnidirectional dielectric mirror fibers," Science, vol. 296, no. 5567, pp. 510-513, 2002.

[111] J. Ballato, T. Hawkins, P. Foy, et al., "Silicon optical fiber," Optics Express, vol. 16, no. 23, pp. 18675-18683, 2008.

[112] D. Webb, in Proceedings of the 1st Asia Pacific Optical Fibre Sensors Conference (APOS '08), Chengdu, China, 2008, invited talk.

[113] C. Zhang, K. Carroll, D. J. Webb, et al., "Recent progress in polymer optical fibre gratings," in Proceedings of the 19th International Conference on Optical Fibre Sensors, vol. 7004 of Proceedings of SPIE, April 2008.

[114] J. M. Yu, X. M. Tao, and H. Y. Tam, "Trans-4stilbenemethanol-doped photosensitive polymer fibers and gratings," Optics Letters, vol. 29, no. 2, pp. 156-158, 2004.

[115] X.-M. Tao, J.-M. Yu, and H.-Y. Tam, "Photosensitive polymer optical fibres and gratings," Transactions of the Institute of Measurement and Control, vol. 29, no. 3-4, pp. 255-270, 2007.

[116] H. Y. Liu, H. B. Liu, G. D. Peng, and P. L. Chu, "Observation of type I and type II gratings behavior in polymer optical fiber," Optics Communications, vol. 220, no. 4-6, pp. 337-343, 2003.

[117] M. S. Milczeswki, H. J. Kalinowski, J. C. C. Silva, J. A. Simões, J. Canning, and C. Martelli, "Determination of orthodontics forces using optic fibre sensors," in Proceedings of the $83 \mathrm{rd}$ Congress of the European Orthodontics Society (EOS '07), Berlin, Germany, 2007.

[118] C. Jewart, K. P. Chen, B. McMillen, et al., "Sensitivity enhancement of fiber Bragg gratings to transverse stress by using microstructural fibers," Optics Letters, vol. 31, no. 15, pp. 2260-2262, 2006. 

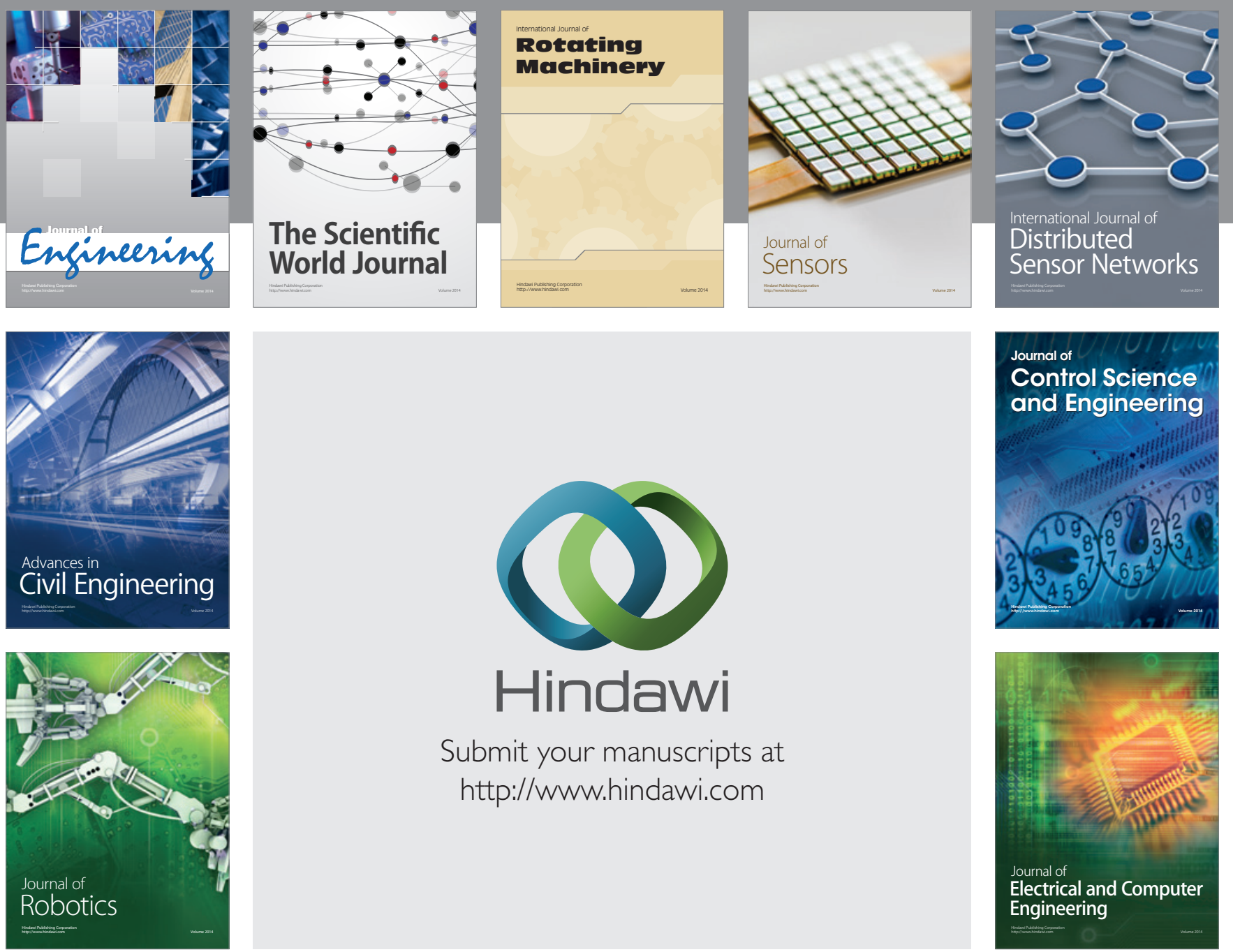

Submit your manuscripts at

http://www.hindawi.com
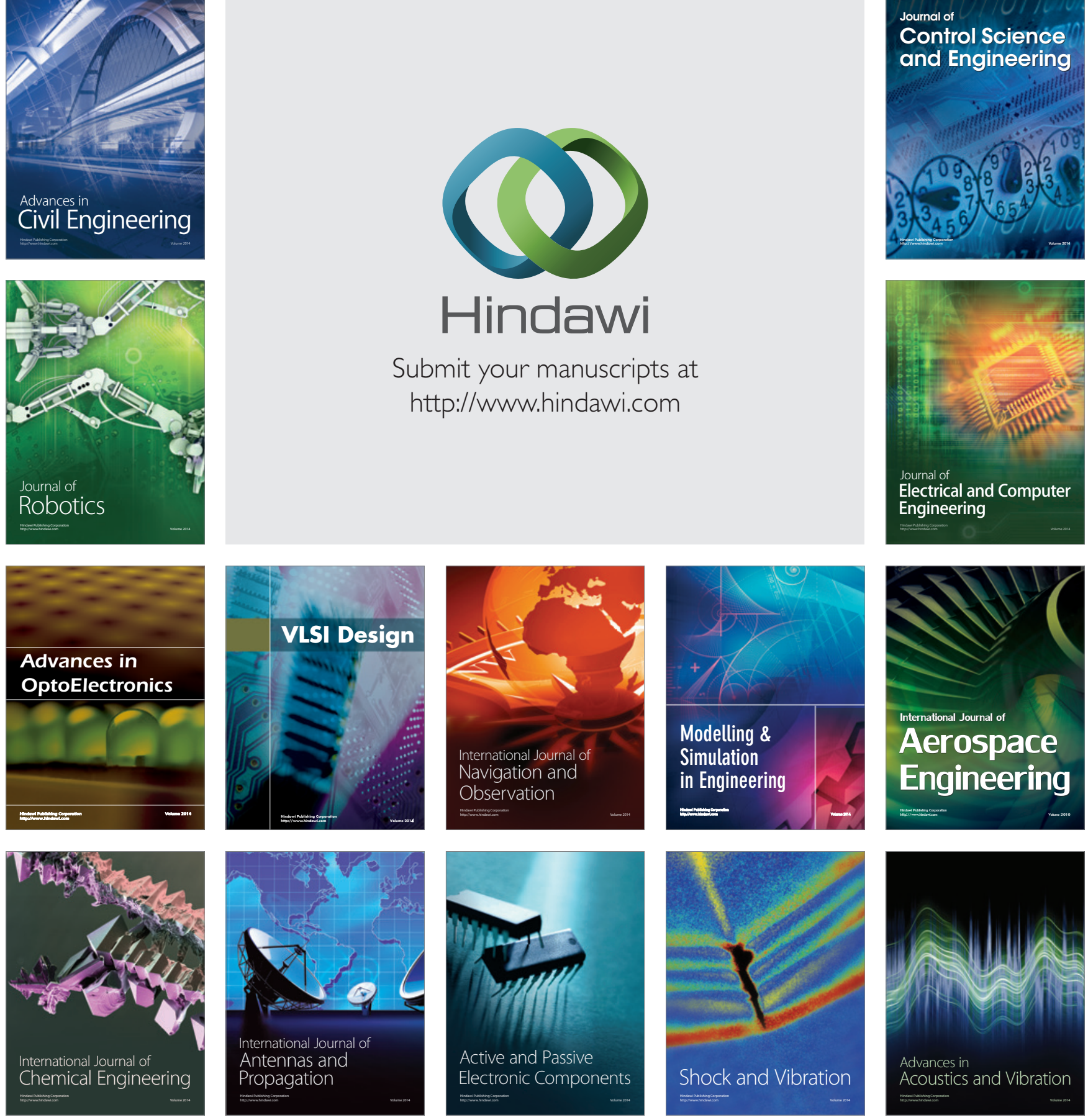